\title{
NMR Characterization of Ten Apple Cultivars from the Piedmont Region
}

\author{
Giacomo Di Matteo ${ }^{1} \mathbb{D}$, Mattia Spano ${ }^{1}\left(\mathbb{D}\right.$, Cristina Esposito $^{2}$, Cristina Santarcangelo $^{2} \mathbb{(}$, Alessandra Baldi $^{3}$, \\ Maria Daglia ${ }^{2,4}\left(\mathbb{C}\right.$, Luisa Mannina $\left.{ }^{1} \mathbb{(}\right)$, Cinzia Ingallina ${ }^{1, *} \mathbb{C}$ and Anatoly P. Sobolev ${ }^{5}$
}

1 Department of Chemistry and Technology of Drugs, Sapienza University of Rome, Piazzale Aldo Moro 5, 00185 Rome, Italy; giacomo.dimatteo@uniroma1.it (G.D.M.); mattia.spano@uniroma1.it (M.S.); luisa.mannina@uniroma1.it (L.M.)

2 Department of Pharmacy, University of Naples Federico II, 80138 Naples, Italy; cristina.esposito@unina.it (C.E.); cristina.santarcangelo@unina.it (C.S.); maria.daglia@unina.it (M.D.)

3 Tefarco Innova, Parco Area delle Scienze 27 / A—Campus, 43124 Parma, Italy; alessandra.baldi.alimenti@gmail.com

4 International Research Center for Food Nutrition and Safety, Jiangsu University, Zhenjiang 212013, China

5 Institute for Biological Systems, Magnetic Resonance Laboratory "Segre-Capitani", CNR, Via Salaria Km 29.300, 00015 Monterotondo, Italy; anatoly.sobolev@cnr.it

* Correspondence: cinzia.ingallina@uniroma1.it

check for updates

Citation: Di Matteo, G.; Spano, M.; Esposito, C.; Santarcangelo, C.; Baldi, A.; Daglia, M.; Mannina, L.; Ingallina, C.; Sobolev, A.P. NMR Characterization of Ten Apple Cultivars from the Piedmont Region. Foods 2021, 10, 289. https://doi.org/ $10.3390 /$ foods 10020289

Academic Editor: Luca Laghi Received: 30 December 2020

Accepted: 25 January 2021

Published: 1 February 2021

Publisher's Note: MDPI stays neutral with regard to jurisdictional claims in published maps and institutional affiliations.

Copyright: (c) 2021 by the authors. Licensee MDPI, Basel, Switzerland. This article is an open access article distributed under the terms and conditions of the Creative Commons Attribution (CC BY) license (https:// creativecommons.org/licenses/by/ $4.0 /)$.

\begin{abstract}
The metabolite profile of ten traditional apple cultivars grown in the Piedmont region (Italy) was studied by means of nuclear magnetic resonance spectroscopy, identifying an overall number of 36 compounds. A more complete assignment of the proton nuclear magnetic resonance $\left({ }^{1} \mathrm{H}\right.$ NMR) resonances from hydroalcoholic and organic apple extracts with respect to literature data was reported, identifying fructose tautomeric forms, galacturonic acid, $\gamma$-aminobutyric acid (GABA), p-coumaroyl moiety, phosphatidylcholine, and digalactosyldiacylglycerol. The chemical profile of each apple cultivar was defined by thorough quantitative NMR analysis of four sugars (fructose, glucose, sucrose, and xylose), nine organic acids (acetic, citric, formic, citramalic, lactic, malic, quinic, and galacturonic acids), six amino acids (alanine, asparagine, aspartate, GABA, isoleucine, and valine), rhamnitol, $p$-coumaroyl derivative, phloretin/phloridzin and choline, as well as $\beta$-sitosterol, fatty acid chains, phosphatidylcholine, and digalactosyldiacylglycerol. Finally, the application of PCA analysis allowed us to highlight possible differences/similarities. The Magnana cultivar showed the highest content of sugars, GABA, valine, isoleucine, and alanine. The Runsé cultivar was characterized by high amounts of organic acids, whereas the Gamba Fina cultivar showed a high content of chlorogenic acid. A significant amount of quinic acid was detected in the Carla cultivar. The knowledge of apple chemical profiles can be useful for industries interested in specific compounds for obtaining ingredients of food supplements and functional foods and for promoting apple valorization and preservation.
\end{abstract}

Keywords: malus domestica; local apple cultivars; NMR; metabolite profile; PCA; food ingredients

\section{Introduction}

Apples are the third most produced fruits in the world [1] after bananas and watermelons, consumed in the human diet as raw or dried products, juice, paste, jam and syrups. From a nutritional point of view, apples are a low-fat (less than $1 \%$ ) and low-protein food (less than 1\%) [2], whereas sugars represent about $10 \%$ of total apple weight, with fructose being the most abundant sugar (6\%). Micronutrients, such as minerals (mainly potassium, phosphorus, calcium, and magnesium), vitamins (mainly vitamin C), and secondary metabolites (such as phenolic compounds [3,4]) are also present. Moreover, apples represent an important source of pectin, a gelling agent obtained from apple pomaces, used for many food industrial productions $[5,6]$. 
In 2018, more than 86 million tons of apples were produced worldwide, with China being the main producer (39 million tons). Italy is the sixth largest producer in the world with 2.4 million tons in 2018 [1]. In many Italian regions, local apple cultivars, used and consumed by the local population, represent an important crop, especially in terms of the local economy. In particular, Alto Adige (912.757 tons in 2020) and Trentino (496.783 tons in 2020) are the most important growing areas of high-quality apples throughout Europe, despite the fact that the cultivation area is not particularly large. However, Piedmont represents today the third largest Italian producer, contributing 225.281 tons of apples to the national production and with an increase of $+13 \%$ with respect to 2019 . Indeed, although Piedmont apple cultivation is not well-known, the story of Piedmont apple growing dates back to the Middle Ages, when the monastic orders cultivated and improved the varieties that had survived barbarian invasions [7-9]. By the early 20th century, Piedmont was home to thousands of varieties, but with the advent of industrial agriculture, decisions with great impact were made regarding selection. The market preferred more productive and attractive apple cultivars, characterized by a bigger size, more attractive appearance, and less delicate flavor, leading to the loss of traditional Italian apple cultivars [10], which survived only in local and niche areas. Today, a turnaround can be observed-the rediscovery of traditional and ancient cultivars that often represent more environmentally sustainable cultivation than commercial ones [11], maintaining both biodiversity and the historical and cultural links [12]. In this scenario, deep characterization and valorization of this heritage is fundamental in order to preserve the special quality of these fruits and to avoid the loss of precious and useful germplasms [12].

Apples' flavor, consistency, taste and, health-related properties strictly depend on the fruit's chemical composition and on the balance between apple component levels. For instance, each organic acid gives rise to a particular acidity sense, and each sugar has its own sweetness level. Apple chemical composition has been largely investigated using targeted chromatographic techniques, such as high-performance liquid chromatography (HPLC) to determine phenolic content [13-16] and gas chromatography (GC) for the determination of sugars, polyols, sugar phosphates, organic acids, sterols, polyphenols [17], fatty acids [18], and other aromatic compounds $[19,20]$. Untargeted proton nuclear magnetic resonance $\left({ }^{1} \mathrm{H}\right.$ NMR) methodologies [21] have focused mainly on apple juice matrixes [19,22,23] to study the chemical composition of new cultivars [24], cultivars with different resistances against fungi attacks [25], and commercial cultivars from Japan and New Zealand [26]. ${ }^{1} \mathrm{H}$ high field NMR spectroscopy has already shown to be a valuable tool to investigate local typical products, such as red sweet peppers [27], white celery [28], tomatoes [29], and olive oils [30]. To date, ancient apple cultivars from Piedmont have been investigated in term of sensory parameters, nutritional aspects [12,31], and genetic characterization [32] through the application of standard analytical techniques. Both Donno et al. and Contessa et al. highlighted superior nutritional traits of ancient Piedmont apple cultivars, with a generally high content of organic acids, sugars, and total phenolic compounds [31]. However, deep chemical characterization by means of advanced analytical methodologies has never been performed.

In this paper, the hydroalcoholic and organic extracts of ten traditional apple cultivars grown in the Piedmont region (northwest Italy) were investigated by means of the NMR methodology to determine their apple metabolite profiles. The application of principal component analysis (PCA) to NMR data allowed us to summarize and highlight the differences and similarities between the selected apple cultivars. Indeed, mathematical tools, better known as chemometrics, are widely used for metabolomic data analysis. In particular, unsupervised methods are utilized to summarize, explore, and discover clusters or trends in the data without a priori attribution of any class membership [33].

The knowledge of chemical apple profile can be extremely important for the introduction of local products in national and international markets and for industries interested in specific apple compounds. 


\section{Materials and Methods}

\subsection{Sampling}

Ten apple cultivars (Malus domestica) typical of Piedmont region, Italy (Figure 1) were collected in the period of their complete maturity, specific for every cultivar, as reported in Table 1. The apples were provided by three farms: "Azienda Agricola Melamangio", "Scuola Malva Arnaldi", and "Azienda Agricola Turaglio". In particular the "Azienda Agricola Melamangio" farm, located in Odalengo Piccolo, provided the variety Canditina (A1); "Scuola Malva Arnaldi", located in Bibiana, provided five varieties: Grigia di Torriana (A2), Magnana (A3), Runsé (A4), Carla (A5) and Gamba Fina (A6); “Azienda Agricola Turaglio", located in Cavour, provided four varieties: Ross Giambon (A7), Dominici (A8), Calvilla (A9) and Grenoble (A10). Some features of the ten cultivars, such as maturity time, size, peel tactility and color, pulp consistency, and color, are reported in [7-9].

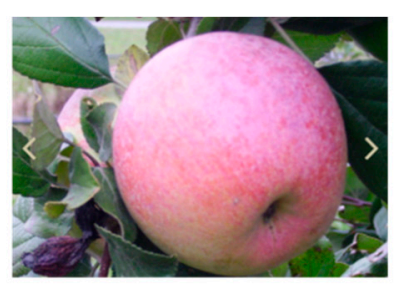

A1

Canditina

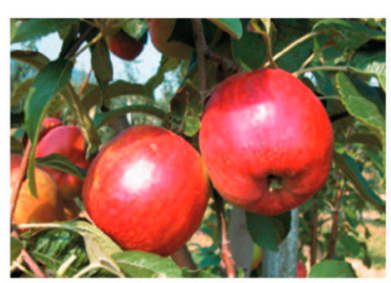

A4

Runsé

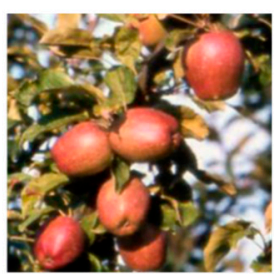

A7

Ross Giambon

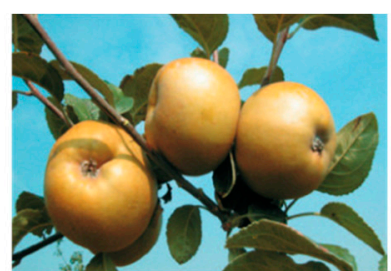

$\mathrm{A} 2$

Grigia di Torriana

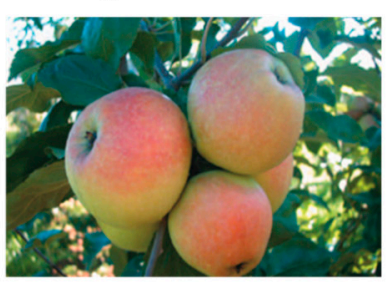

A5

Carla

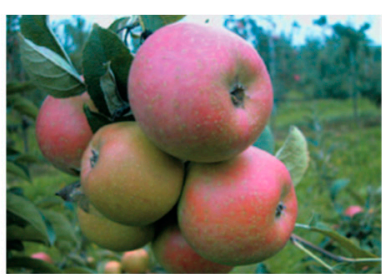

A3

Magnana

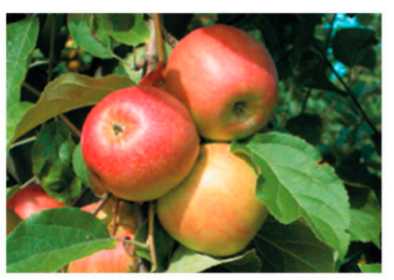

A6

Gamba Fina

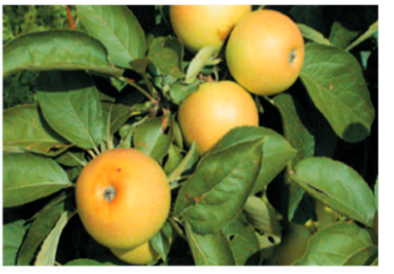

A8

Dominici

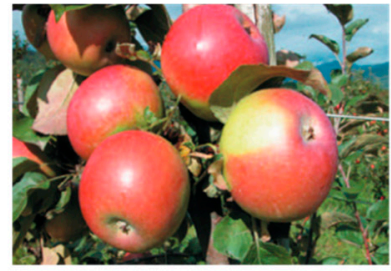

A9

Calvilla

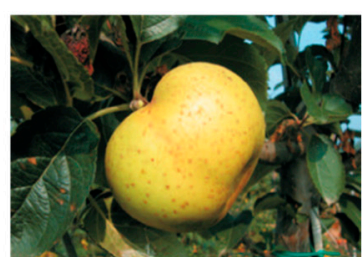

A10

Grenoble

Figure 1. Apple cultivars from the Piedmont region.

\subsection{Chemicals}

Deuterated water $\left(\mathrm{D}_{2} \mathrm{O}\right) 99.97 \% \mathrm{D}$, methanol-D4 99.80\% D, chloroform-D 99.80\% D + $0.03 \%$ tetramethylsilane (TMS), and 3-(trimethylsilyl)-propionic-2,2,3,3-d4 acid sodium salt (TSP) were purchased from Euriso-Top (Saclay, France). Anhydrous potassium phosphate dibasic $\left(\mathrm{K}_{2} \mathrm{HPO}_{4}\right)$ and anhydrous potassium phosphate monobasic $\left(\mathrm{KH}_{2} \mathrm{PO}_{4}\right)$ were purchased from Sigma-Aldrich (St. Louis, MO, USA). Methanol (HPLC-grade) and chloroform (HPLC-grade) were purchased from Carlo Erba Reagenti (Milan, Italy). Millipore grade water was purchased from Tito Menichelli S.r.l. (Rome, Italy). 
Table 1. Characteristics (maturity time, peel, pulp and size) of the ten investigated apple cultivars [7-9,34].

\begin{tabular}{|c|c|c|c|c|c|}
\hline $\mathbf{N}^{\circ}$ & Cultivar & Maturity Time & Peel & Pulp & Size \\
\hline A1 & Canditina & Late October & $\begin{array}{c}\text { Smooth, green with red } \\
\text { overcolour }\end{array}$ & $\begin{array}{c}\text { Juicy, } \\
\text { white/green }\end{array}$ & Medium \\
\hline A2 & $\begin{array}{l}\text { Grigia di } \\
\text { Torriana }\end{array}$ & Mid/late October & Rough, green & Soft, white & Medium \\
\hline A3 & Magnana & $\begin{array}{c}\text { Late October/early } \\
\text { November }\end{array}$ & $\begin{array}{c}\text { Smooth/rough, } \\
\text { white/green with red } \\
\text { overcolour }\end{array}$ & $\begin{array}{c}\text { Soft, } \\
\text { white/green }\end{array}$ & Medium \\
\hline $\mathrm{A} 4$ & Runsé & $\begin{array}{c}\text { Late October/early } \\
\text { November }\end{array}$ & Smooth, red & $\begin{array}{c}\text { Juicy, } \\
\text { white/pink }\end{array}$ & Medium \\
\hline A5 & Carla & Mid/late September & $\begin{array}{l}\text { Smooth, yellow with } \\
\text { red/orange overcolour }\end{array}$ & Soft, white & Medium/small \\
\hline A6 & Gamba Fina & Early/Mid October & $\begin{array}{l}\text { Smooth, yellow/green } \\
\text { with red overcolour }\end{array}$ & Soft, white & Medium/small \\
\hline A7 & Ross Giambon & Mid October & Smooth, yellow / green & Juicy, white & Large \\
\hline A8 & Dominici & Mid October & $\begin{array}{l}\text { Rough, yellow/green with } \\
\text { red overcolour }\end{array}$ & Crisp, white & Large \\
\hline A9 & Calvilla & Mid October & $\begin{array}{c}\text { Smooth, green with red } \\
\text { overcolour }\end{array}$ & $\begin{array}{l}\text { Crisp and juicy, } \\
\text { white/green }\end{array}$ & Medium \\
\hline A10 & Grenoble & Late October & Rough, green & $\begin{array}{c}\text { Crisp, } \\
\text { white/green }\end{array}$ & Small \\
\hline
\end{tabular}

\subsection{Sample Preparation}

In the sample preparation step, apple skin and pulp and seeds were removed. Seven apples were sampled for each cultivar. The apples were first washed with water and $\mathrm{Na}_{2} \mathrm{CO}_{3}$ to eliminate every dirt residue. A clove was taken from each apple and subsequently shredded with a ceramic knife. To control the oxidation during preparation, the samples were cut in an ice bath. All the samples were transferred to the falcon and lyophilized for 7 days. All apples were cut into eight parts, and seven pieces (one from each apple of the cultivar) were chopped together into smaller pieces. Each sample was then freeze-dried and pulverized with mortar and pestle. The freeze-drying process promotes water removal, thereby reducing the oxidation process.

\subsection{Extraction Procedure for NMR Analysis}

Extracts for NMR analysis were obtained following the protocol previously described [35] with some modifications. Powder $(0.5 \mathrm{~g})$ was sequentially added with a $3 \mathrm{~mL}$ methanol/chloroform (2:1 $\mathrm{v} / \mathrm{v})$ mixture, containing $1 \mathrm{~mL}$ of chloroform and $1.8 \mathrm{~mL}$ of Millipore-grade water, which was shaken slightly after each solvent addition. The obtained emulsion was stored at $4{ }^{\circ} \mathrm{C}$ for $40 \mathrm{~min}$ and then centrifuged at $4200 \times \mathrm{g}$ for $15 \mathrm{~min}$ at $4{ }^{\circ} \mathrm{C}$. The organic and hydroalcoholic phases were separated, and the residual pellet was extracted again using half of the solvent volumes to ensure complete extraction of the soluble metabolites. A slow $\mathrm{N}_{2}$ flow was used for drying. The obtained extracts were stored at $-20{ }^{\circ} \mathrm{C}$ until analysis.

\subsection{Metabolic Profile by NMR Analysis}

Hydroalcoholic and organic extracts were analyzed using a Bruker AVANCE 600 spectrometer (Bruker, Milan, Italy) at $28{ }^{\circ} \mathrm{C}$ operating at $600.13 \mathrm{MHz}$ (proton frequency) and equipped with a Bruker multinuclear z-gradient $5 \mathrm{~mm}$ probe head. The temperature for NMR spectral analysis $\left(28^{\circ} \mathrm{C}\right)$ was chosen according to the internal laboratory protocol that assures temperature maintenance within $\left( \pm 0.1^{\circ} \mathrm{C}\right)$ limits and is close to the experimental conditions reported in most databases. Each dried hydroalcoholic extract was solubilized in $1 \mathrm{~mL}$ of $\mathrm{D}_{2} \mathrm{O} ; 0.2 \mathrm{~mL}$ of the obtained solution was mixed with $0.5 \mathrm{~mL}$ of $400 \mathrm{mM}$ phosphate buffer $(\mathrm{pH} 7.4) / \mathrm{D}_{2} \mathrm{O}$ containing $2 \mathrm{mM}$ solution of TSP (internal standard) and transferred into a $5 \mathrm{~mm}$ NMR tube. The use of TPS as internal standard 
does not interfere with the protein in apple extracts, as the balk of apple proteins is not easily soluble in water [36]. ${ }^{1} \mathrm{H}$ spectra (Bruker pulse sequence zgpr) of hydroalcoholic extracts were acquired with 200 transients, a recycle delay of $5 \mathrm{~s}$, an acquisition time of $2.28 \mathrm{~s}$, a $90^{\circ}$ flip angle pulse of $14 \mu \mathrm{s}$, and $32 \mathrm{~K}$ data points. The water signal was suppressed using solvent pre-saturation. Each dried organic extract was solubilized in $0.7 \mathrm{~mL}$ of the $\mathrm{CDCl}_{3} / \mathrm{CD}_{3} \mathrm{OD}(2: 1 \mathrm{v} / \mathrm{v})$ mixture and transferred into a $5 \mathrm{~mm}$ NMR tube that was flame sealed. ${ }^{1} \mathrm{H}$ spectra (Bruker pulse sequence $z g$ ) of the organic extracts were acquired with 128 transients, a recycle delay of $5 \mathrm{~s}$, an acquisition time of $1.82 \mathrm{~s}$, a $90^{\circ}$ flip angle pulse of $10.5 \mu \mathrm{s}$, and $32 \mathrm{~K}$ data points.

Two-dimensional (2D) NMR experiments $\left({ }^{1} \mathrm{H}^{-1} \mathrm{H}\right.$ TOtal Correlated SpectroscopY (TOCSY), ${ }^{1} \mathrm{H}_{-}{ }^{13} \mathrm{C}$ Heteronuclear Single Quantum Coherence (HSQC), and ${ }^{1} \mathrm{H}_{-}{ }^{13} \mathrm{C}$ Heteronuclear Multiple Bond Correlation (HMBC) were performed on each hydroalcoholic and organic extract under the experimental conditions previously reported [29].

In order to evaluate the repeatability of the protocol and to ensure the complete extraction of the soluble metabolites, all dry matter after freeze-drying was recovered, and the entire procedure (from the extraction to NMR analysis) was carried out in triplicate.

In Table 2, the signals of the identified metabolites in the hydroalcoholic extracts ${ }^{1} \mathrm{H}$ NMR spectra are reported. Among them, the 26 selected signals marked with asterisks were integrated using the Bruker TOPSPIN 1.3 software and normalized with respect to the methyl group signal of TSP $(0.00 \mathrm{ppm})$, set to 100 . The quantified metabolites were expressed in $\mathrm{mg} / 100 \mathrm{~g}$ of the dried sample $\pm \mathrm{SD}$ (standard deviation) (Table S1).

Table 2. Metabolites identified in the $600.13 \mathrm{MHz}$ proton nuclear magnetic resonance $\left({ }^{1} \mathrm{H} \mathrm{NMR}\right),{ }^{1} \mathrm{H}-$ ${ }^{1} \mathrm{H}$ TOCSY, ${ }^{1} \mathrm{H}-{ }^{13} \mathrm{C}$ HSQC, and ${ }^{1} \mathrm{H}-{ }^{13} \mathrm{C}$ HMBC spectra of Bligh-Dyer hydroalcoholic extracts of apple in $0.7 \mathrm{~mL}$ of phosphate buffer $/ \mathrm{D}_{2} \mathrm{O}$ at $28^{\circ} \mathrm{C}$. Asterisks $\left(^{*}\right)$ indicate signals selected for integration.

\begin{tabular}{|c|c|c|c|c|}
\hline Compound & Assignment & ${ }^{1} \mathrm{H}(\mathrm{ppm})$ & $\begin{array}{l}\text { Multiplicity } \\
(\mathrm{J}(\mathbf{H z}))\end{array}$ & ${ }^{13} \mathrm{C}(\mathrm{ppm})$ \\
\hline \multicolumn{5}{|c|}{ Carbohydrates } \\
\hline \multirow[t]{4}{*}{$\alpha$-D-Fructofuranose } & C-2 & & & 105.5 \\
\hline & $\mathrm{CH}-3$ & 4.13 * & & 83.0 \\
\hline & $\mathrm{CH}-4$ & 4.00 & & 77.2 \\
\hline & $\mathrm{CH}-5$ & 4.07 & & 82.4 \\
\hline \multirow[t]{5}{*}{$\beta$-D-Fructofuranose } & C-2 & & & 102.6 \\
\hline & $\mathrm{CH}-3$ & 4.12 * & & 76.6 \\
\hline & $\mathrm{CH}-4$ & 4.12 * & & 75.5 \\
\hline & $\mathrm{CH}-5$ & 3.84 & & 81.7 \\
\hline & $\mathrm{CH} 2-6,6^{\prime}$ & $3.83 ; 3.68$ & & \\
\hline \multirow[t]{6}{*}{$\beta$-D-Fructopyranose } & $\mathrm{CH}_{2}-1,1^{\prime}$ & $3.57 ; 3.72$ & & 64.9 \\
\hline & C-2 & & & 99.2 \\
\hline & $\mathrm{CH}-3$ & 3.80 & & 68.6 \\
\hline & $\mathrm{CH}-4$ & 3.90 & & 70.7 \\
\hline & $\mathrm{CH}-5$ & 4.01 & & 70.2 \\
\hline & $\mathrm{CH}_{2}-6,6^{\prime}$ & $3.72 ; 4.03$ & & 64.5 \\
\hline \multirow[t]{3}{*}{$\alpha-X y l o s e$} & $\mathrm{CH}-1$ & $5.20 *$ & $\mathrm{~d}^{\mathrm{a}}(3.8)$ & 93.4 \\
\hline & $\mathrm{CH}-2$ & 3.55 & & \\
\hline & $\mathrm{CH}-3$ & 3.65 & & \\
\hline \multirow[t]{3}{*}{$\beta-$ Xylose } & $\mathrm{CH}-1$ & $4.59 *$ & $\mathrm{~d}(8.0)$ & 97.7 \\
\hline & $\mathrm{CH}-2$ & 3.54 & & \\
\hline & $\mathrm{CH}-3$ & 3.69 & & \\
\hline \multirow[t]{5}{*}{$\alpha$-Glucose } & $\mathrm{CH}-1$ & $5.24 *$ & $\mathrm{~d}(3.8)$ & 93.2 \\
\hline & $\mathrm{CH}-2$ & 3.55 & & 72.2 \\
\hline & $\mathrm{CH}-3$ & 3.73 & & 73.7 \\
\hline & $\mathrm{CH}-4$ & 3.42 & & 70.3 \\
\hline & $\mathrm{CH}-5$ & 3.84 & & 72.8 \\
\hline$\beta$-Glucose & $\mathrm{CH}-1$ & $4.66 *$ & $\mathrm{~d}(8.0)$ & 97.0 \\
\hline
\end{tabular}


Table 2. Cont.

\begin{tabular}{|c|c|c|c|c|}
\hline Compound & Assignment & ${ }^{1} \mathrm{H}(\mathrm{ppm})$ & $\begin{array}{l}\text { Multiplicity } \\
(\mathrm{J}(\mathrm{Hz}))\end{array}$ & ${ }^{13} \mathrm{C}(\mathrm{ppm})$ \\
\hline \multirow{10}{*}{ Sucrose } & $\mathrm{CH}-2$ & 3.25 & $\mathrm{dd}^{\mathrm{b}}(9.4 ; 7.9)$ & 75.1 \\
\hline & $\mathrm{CH}-3$ & 3.51 & & 76.9 \\
\hline & $\mathrm{CH}-4$ & 3.43 & & 70.7 \\
\hline & CH-5 & 3.49 & & 76.9 \\
\hline & $\mathrm{CH}_{2}-6,6^{\prime}$ & $3.90 ; 3.75$ & & 61.8 \\
\hline & $\begin{array}{c}\mathrm{CH}-1 \\
\text { (glucose) }\end{array}$ & $5.42 *$ & $\mathrm{~d}(3.8)$ & 93.3 \\
\hline & $\mathrm{CH}-2$ & 3.57 & & 72.0 \\
\hline & $\mathrm{CH}-3$ & 3.77 & & 73.7 \\
\hline & $\mathrm{CH}-4$ & 3.47 & & 70.1 \\
\hline & CH-5 & 3.85 & & 73.5 \\
\hline \multirow{5}{*}{ Rhamnitol } & C-2 (fructose) & & & 104.8 \\
\hline & $\mathrm{CH}-3$ & 4.22 & $\mathrm{~d}(8.8)$ & 77.3 \\
\hline & $\mathrm{CH}_{3}$ & $1.28 *$ & $\mathrm{~d}(6.4)$ & 20.0 \\
\hline & $\mathrm{CH}-2$ & 3.88 & & 68.2 \\
\hline & $\mathrm{CH}-3$ & 3.61 & & 74.3 \\
\hline \multicolumn{5}{|c|}{ Organic acids } \\
\hline Acetic acid & $\alpha-\mathrm{CH}_{3}$ & $1.92 *$ & $\mathrm{~S}^{\mathrm{c}}$ & \\
\hline \multirow[t]{5}{*}{ Citric acid } & $\alpha, \gamma-\mathrm{CH}$ & $2.54 *$ & $\mathrm{~d}(15.2)$ & 46.7 \\
\hline & $\alpha^{\prime}, \gamma^{\prime}-\mathrm{CH}$ & 2.67 & & 46.7 \\
\hline & $\beta-C$ & & & 76.5 \\
\hline & $1,5-\mathrm{COOH}$ & & & 180.8 \\
\hline & $6-\mathrm{COOH}$ & & & 183.0 \\
\hline Formic acid & $\mathrm{HCOOH}$ & $8.46^{*}$ & $\mathrm{~s}$ & \\
\hline \multirow{5}{*}{ Citramalic acid } & $\beta-\mathrm{CH}_{3}$ & 1.33 * & $\mathrm{s}$ & 26.5 \\
\hline & $\beta-\mathrm{CH}_{2}$ & 2.44 & $\mathrm{~d}(15.8)$ & 47.7 \\
\hline & $\beta^{\prime}-\mathrm{CH}_{2}$ & 2.74 & $\mathrm{~d}(15.8)$ & 47.7 \\
\hline & $\alpha-C^{2}$ & & & 75.5 \\
\hline & $1,4-\mathrm{COOH}$ & & & 184.2 \\
\hline \multirow[t]{3}{*}{ Lactic acid } & $\beta-\mathrm{CH}_{3}$ & $1.33 *$ & $\mathrm{~d}(7.0)$ & 21.4 \\
\hline & $\alpha-\mathrm{CH}$ & 4.12 & & 69.8 \\
\hline & $\mathrm{COOH}$ & & & 183.4 \\
\hline \multirow[t]{3}{*}{ Malic acid } & $\alpha-\mathrm{CH}$ & $4.30 *$ & $\mathrm{dd}(9.9 ; 3.2)$ & 71.6 \\
\hline & $\beta-\mathrm{CH}$ & 2.67 & dd $(15.4 ; 3.2)$ & 43.9 \\
\hline & $\beta^{\prime}-\mathrm{CH}$ & 2.37 & dd $(15.4 ; 9.9)$ & 43.9 \\
\hline \multirow[t]{5}{*}{ Quinic acid } & $C-1$ & & & 78.3 \\
\hline & $\mathrm{CH}_{2}-2,2^{\prime}$ & $1.88 * ; 2.06$ & $\begin{array}{l}\mathrm{dd}(13.5 ; \\
10.8) ; \mathrm{m}\end{array}$ & 41.9 \\
\hline & $\mathrm{CH}-3$ & 4.03 & $\mathrm{~m}^{\mathrm{d}}$ & 68.0 \\
\hline & $\mathrm{CH}-4$ & 3.57 & $\mathrm{~m}$ & 76.2 \\
\hline & $\mathrm{CH}-5$ & 4.16 & $\mathrm{~m}$ & 71.3 \\
\hline \multirow[t]{6}{*}{$\alpha$-Galacturonic acid } & $\mathrm{CH}-1$ & 5.31 & $\mathrm{~d}(3.8)$ & 93.1 \\
\hline & $\mathrm{CH}-2$ & 3.80 & & \\
\hline & $\mathrm{CH}-3$ & 3.90 & & \\
\hline & $\mathrm{CH}-4$ & 4.29 & & \\
\hline & $\mathrm{CH}-5$ & $4.41^{*}$ & $\mathrm{~d}(1.2)$ & 72.5 \\
\hline & $\mathrm{COOH}$ & & & 177.1 \\
\hline \multicolumn{5}{|c|}{ Amino acids } \\
\hline \multirow[t]{3}{*}{ Alanine } & $\alpha-\mathrm{CH}$ & 3.80 & & 51.5 \\
\hline & $\beta-\mathrm{CH}_{3}$ & 1.49 * & $\mathrm{d}(7.3)$ & 17.3 \\
\hline & $\mathrm{COOH}$ & & & 176.8 \\
\hline \multirow[t]{3}{*}{ Asparagine } & $\alpha-\mathrm{CH}$ & 4.05 & & 52.3 \\
\hline & $\beta, \beta^{\prime}-\mathrm{CH}_{2}$ & $2.88 * ; 2.96$ & $\begin{array}{l}\text { dd }(7.4 ; 16.9) \\
\text { dd }(4.3 ; 12.6)\end{array}$ & 35.6 \\
\hline & $\mathrm{COOH}$ & & & 175.5 \\
\hline Aspartate & $\beta, \beta^{\prime}-\mathrm{CH}_{2}$ & $2.70 ; 2.81$ * & $\mathrm{dd}(3.7 ; 17.4)$ & \\
\hline$\gamma$-Aminobutyrate & $\alpha-\mathrm{CH}_{2}$ & $2.30 *$ & $\mathrm{t}^{\mathrm{e}}(7.4)$ & 35.3 \\
\hline
\end{tabular}


Table 2. Cont.

\begin{tabular}{|c|c|c|c|c|}
\hline Compound & Assignment & ${ }^{1} \mathrm{H}(\mathrm{ppm})$ & $\begin{array}{l}\text { Multiplicity } \\
(\mathrm{J}(\mathrm{Hz}))\end{array}$ & ${ }^{13} \mathrm{C}$ (ppm) \\
\hline & $\beta-\mathrm{CH}_{2}$ & 1.90 & & 24.7 \\
\hline & $\gamma-\mathrm{CH}_{2}$ & 3.01 & & \\
\hline Isoleucine & $\gamma-\mathrm{CH}_{3}$ & $1.01 *$ & $\mathrm{~d}(7.1)$ & \\
\hline \multirow[t]{3}{*}{ Valine } & $\gamma-\mathrm{CH}_{3}$ & $0.99 *$ & $\mathrm{~d}(7.1)$ & \\
\hline & $\gamma^{\prime}-\mathrm{CH}_{3}$ & 1.05 & $\mathrm{~d}(7.1)$ & \\
\hline & Miscell & etabolites & & \\
\hline \multirow[t]{9}{*}{ Chlorogenic acid } & $\mathrm{CH}_{2}-2$ & 2.20 & & \\
\hline & $\mathrm{CH}-3$ & 5.33 & $\mathrm{~m}$ & 72.2 \\
\hline & $\mathrm{CH}_{2}-6$ & 2.04 & & \\
\hline & $\mathrm{CH}-1^{\prime}$ & $6.42 *$ & $\mathrm{~d}(16.0)$ & 115.8 \\
\hline & $\mathrm{CH}-2^{\prime}$ & 7.67 & $\mathrm{~d}(16.0)$ & 147.3 \\
\hline & $\mathrm{CH}-3^{\prime}$ & 7.22 & $\mathrm{~d}(2.0)$ & 116.3 \\
\hline & $\mathrm{CH}-6^{\prime}$ & 6.98 & & 116.7 \\
\hline & CH-7' & 7.15 & & 123.8 \\
\hline & $\mathrm{CH}_{2}-6,6^{\prime}$ & $1.97 ; 2.05$ & $\mathrm{~m}$ & 38.5 \\
\hline \multirow{4}{*}{$\begin{array}{c}\text { Choline } \\
p \text {-Coumaric acid } \\
\text { derivative }\end{array}$} & $\mathrm{N}\left(\mathrm{CH}_{3}\right)_{3}+$ & $3.21 *$ & $\mathrm{~s}$ & 55.1 \\
\hline & $\mathrm{CH}-2,6$ & $7.62 *$ & $\mathrm{~d}(8.8)$ & 131.9 \\
\hline & $\mathrm{CH}-3,5$ & 6.97 & & \\
\hline & $\mathrm{CH}=\mathrm{CH}$ & $7.79 ; 6.51$ & $\mathrm{~d}(16.1)$ & \\
\hline \multirow[t]{4}{*}{ Phloretin/Phloridzin } & $\mathrm{CH}-2,6$ & 7.16 & $\mathrm{~d}(8.3)$ & 130.7 \\
\hline & $\mathrm{CH}-3,5$ & $6.85 *$ & $\mathrm{~d}(8.3)$ & 116.5 \\
\hline & $\mathrm{CH}-3^{\prime}, 5^{\prime}$ & $6.19 ; 6.24$ & $\mathrm{~s}$ & $96.6 ; 97.3$ \\
\hline & $\beta-\mathrm{CH}_{2}$ & 2.92 & & 31.0 \\
\hline
\end{tabular}

${ }^{\mathrm{a}} \mathrm{d}=$ doublet; $^{\mathrm{b}} \mathrm{dd}=$ double doublet; $^{\mathrm{c}} \mathrm{s}=$ singlet; $^{\mathrm{d}} \mathrm{m}=$ multiplet; $^{\mathrm{e}} \mathrm{t}=$ triplet.

The integral areas of the 7 selected signals in the ${ }^{1} \mathrm{H}$ NMR spectra of organic extracts (Table 3) were measured using the Bruker TOPSPIN 1.3 software and normalized with respect to the integral ( $\left.\mathrm{I}_{\mathrm{FA}}\right)$ of the $\alpha-\mathrm{CH}_{2}$ group signal of all fatty acids $(2.30 \mathrm{ppm})$, set to 100 . The molar $\%$ values \pm SD of fatty acids, $\beta$-sitosterol, phosphatidylcholine, and digalactosyldiacylglycerol were calculated with consideration of the number of equivalent protons using the following equations:

$$
\begin{gathered}
\%_{\mathrm{STE}}=100\left(0.66 \mathrm{I}_{\mathrm{STE}} / \mathrm{I}_{\mathrm{tot}}\right) \\
\%_{\mathrm{TRI}}=100\left(0.5 \mathrm{I}_{\mathrm{TRI}} / \mathrm{I}_{\mathrm{tot}}\right) \\
\%_{\mathrm{DI}}=100\left(\mathrm{I}_{\mathrm{DI}} / \mathrm{I}_{\mathrm{tot}}\right) \\
\%_{\mathrm{MONO}}=100\left(\mathrm{I}_{\mathrm{UNS}}-2 \mathrm{I}_{\mathrm{DI}}-1.5 \mathrm{I}_{\mathrm{TRI}}\right) / \mathrm{I}_{\mathrm{tot}} \\
\%_{\mathrm{SAT}}=100\left(\mathrm{I}_{\mathrm{FA}}-\mathrm{I}_{\mathrm{DI}}-0.5 \mathrm{I}_{\mathrm{TRI}}-\%_{\mathrm{MONO}}\right) / \mathrm{I}_{\mathrm{tot}} \\
\%_{\mathrm{PC}}=100\left(4 \mathrm{I}_{\mathrm{PC}} / 9 \mathrm{I}_{\mathrm{tot}}\right) \\
\%_{\mathrm{DGDG}}=100\left(4 \mathrm{I}_{\mathrm{DGDG}} / \mathrm{I}_{\mathrm{tot}}\right)
\end{gathered}
$$

where $\%_{\text {STE }}$, $\%_{\text {TRI }}, \%_{\mathrm{DI}}, \%_{\mathrm{MONO}}, \%_{\mathrm{SAT}}, \%_{\mathrm{PC}}$, and $\%_{\mathrm{DGDG}}$ are the molar $\%$ of $\beta$-sitosterol, triunsaturated fatty acids, di-unsaturated fatty acids, mono-unsaturated fatty acids, saturated fatty acids, phosphatidylcholine, and digalactosyldiacylglycerol, respectively. ISTE, IRI, $\mathrm{I}_{\mathrm{DI}}, \mathrm{I}_{\mathrm{UNS}}, \mathrm{I}_{\mathrm{FA}}, \mathrm{I}_{\mathrm{PC}}$, and $\mathrm{I}_{\mathrm{DGDG}}$ are integrals, whereas $\mathrm{I}_{\mathrm{tot}}$ is calculated according to the following equation:

$$
\mathrm{I}_{\mathrm{tot}}=\mathrm{I}_{\mathrm{FA}}+0.66 \mathrm{I}_{\mathrm{STE}}
$$


Table 3. Metabolites identified in the $600.13 \mathrm{MHz}{ }^{1} \mathrm{H}$ NMR, ${ }^{1} \mathrm{H}_{-}{ }^{1} \mathrm{H}$ TOCSY, ${ }^{1} \mathrm{H}_{-}{ }^{13} \mathrm{C}$ HSQC, and ${ }^{1} \mathrm{H}_{-}{ }^{13} \mathrm{C}$ HMBC spectra $\left(28{ }^{\circ} \mathrm{C}\right)$ of Bligh-Dyer organic extracts of apple in $\mathrm{CDCl}_{3} / \mathrm{CD}_{3} \mathrm{OD}(2: 1 v / v)$ mixture at $28{ }^{\circ} \mathrm{C}$. Asterisks $\left(^{*}\right)$ indicate signals selected for integration. For the integration of total fatty acids $\left(\mathrm{I}_{\mathrm{FA}}\right)$, the region of $2.22-2.35$ was considered. For the integration of total unsaturated fatty acids ( $\mathrm{I}_{\mathrm{UNS}}$ ), the region of 5.25-5.384 was considered.

\begin{tabular}{|c|c|c|c|c|}
\hline Compound & Assignment & ${ }^{1} \mathbf{H}(\mathrm{ppm})$ & $\begin{array}{l}\text { Multiplicity } \\
(\mathrm{J}(\mathrm{Hz}))\end{array}$ & ${ }^{13} \mathrm{C}(\mathrm{ppm})$ \\
\hline Oleic fatty chain & $\mathrm{COO}$ & & & 174.4 \\
\hline \multirow[t]{10}{*}{$\left(\mathrm{C} 18: 1 \Delta^{9}\right)$} & $\mathrm{CH}_{2}-2$ & 2.30 & & 34.6 \\
\hline & $\mathrm{CH}_{2}-3$ & 1.58 & $\mathrm{~m}^{\mathrm{a}}$ & 25.3 \\
\hline & $\mathrm{CH}_{2}-4,7$ & 1.30 & $\mathrm{~m}$ & 29.5 \\
\hline & $\mathrm{CH}_{2}-8$ & 2.01 & $\mathrm{~m}$ & 27.6 \\
\hline & $\mathrm{CH}=\mathrm{CH} 9,10$ & 5.31 & $\mathrm{~m}$ & 130.6 \\
\hline & $\mathrm{CH}_{2}-11$ & 2.01 & $\mathrm{~m}$ & 27.6 \\
\hline & $\mathrm{CH}_{2}-12,15$ & $1.33-1.30$ & $\mathrm{~m}$ & $29.4-30.2$ \\
\hline & $\mathrm{CH}_{2}-16$ & 1.28 & $\mathrm{~m}$ & 31.6 \\
\hline & $\mathrm{CH}_{2}-17$ & 1.26 & $\mathrm{~m}$ & 23.0 \\
\hline & $\mathrm{CH}_{3}-18$ & 0.84 & $t^{b}$ & 14.4 \\
\hline \multirow{15}{*}{$\begin{array}{l}\text { Linoleic fatty chain } \\
\quad\left(\mathrm{C} 18: 2 \Delta^{9,12}\right)\end{array}$} & $\mathrm{COO}$ & & & 174.4 \\
\hline & $\mathrm{CH}_{2}-2$ & 2.30 & & 34.6 \\
\hline & $\mathrm{CH}_{2}-3$ & 1.58 & $\mathrm{~m}$ & 25.3 \\
\hline & $\mathrm{CH}_{2}-4,7$ & $1.32-1.28$ & $\mathrm{~m}$ & 29.5 \\
\hline & $\mathrm{CH}_{2}-8$ & 2.02 & $\mathrm{~m}$ & 27.6 \\
\hline & $\mathrm{CH}=9$ & 5.34 & $\mathrm{~m}$ & 130.6 \\
\hline & $\mathrm{CH}=10$ & 5.31 & $\mathrm{~m}$ & 128.6 \\
\hline & $\mathrm{CH}_{2}-11$ & $2.73 *\left(\mathrm{I}_{\mathrm{DI}}\right)$ & $\mathrm{t}(6.8)$ & 26.0 \\
\hline & $\mathrm{CH}=12$ & 5.31 & $\mathrm{~m}$ & 128.6 \\
\hline & $\mathrm{CH}=13$ & 5.34 & $\mathrm{~m}$ & 130.6 \\
\hline & $\mathrm{CH}_{2}-14$ & 2.02 & $\mathrm{~m}$ & 27.6 \\
\hline & $\mathrm{CH}_{2}-15$ & 1.29 & $\mathrm{~m}$ & 29.4 \\
\hline & $\mathrm{CH}_{2}-16$ & 1.29 & $\mathrm{~m}$ & 31.6 \\
\hline & $\mathrm{CH}_{2}-17$ & 1.23 & $\mathrm{~m}$ & 23.0 \\
\hline & $\mathrm{CH}_{3}-18$ & 0.85 & $\mathrm{t}$ & 14.4 \\
\hline \multirow{14}{*}{$\begin{array}{l}\text { Linolenic fatty chain } \\
\quad\left(\mathrm{C} 18: 3 \Delta^{9,12,15}\right)\end{array}$} & $\mathrm{COO}$ & & & 174.4 \\
\hline & $\mathrm{CH}_{2}-2$ & 2.30 & & 34.9 \\
\hline & $\mathrm{CH}_{2}-3$ & 1.58 & $\mathrm{~m}$ & 25.3 \\
\hline & $\mathrm{CH}_{2}-4,7$ & 1.30 & $\mathrm{~m}$ & 29.5 \\
\hline & $\mathrm{CH}_{2}-8$ & 2.03 & $\mathrm{~m}$ & 27.6 \\
\hline & $\mathrm{CH}=9$ & 5.34 & $\mathrm{~m}$ & 130.6 \\
\hline & $\mathrm{CH}=10$ & 5.30 & $\mathrm{~m}$ & 128.6 \\
\hline & $\mathrm{CH}_{2} 11$ & $2.77 *\left(\mathrm{I}_{\mathrm{TRI}}\right)$ & $t(6.2)$ & 26.0 \\
\hline & $\mathrm{CH}=\mathrm{CH} 12,13$ & 5.30 & $\mathrm{~m}$ & 128.6 \\
\hline & $\mathrm{CH}_{2}-14$ & $2.77 *\left(\mathrm{I}_{\mathrm{TRI}}\right)$ & $t(6.2)$ & 26.0 \\
\hline & $\mathrm{CH}=15$ & 5.28 & $\mathrm{~m}$ & 127.4 \\
\hline & $\mathrm{CH}=16$ & 5.34 & $\mathrm{~m}$ & 132.2 \\
\hline & $\mathrm{CH}_{2}-17$ & 2.03 & $\mathrm{~m}$ & 20.9 \\
\hline & $\mathrm{CH}_{3}-18$ & 0.94 & $\mathrm{t}(7.6)$ & 14.4 \\
\hline \multirow[t]{6}{*}{ Saturated fatty acids } & $\mathrm{COO}$ & & & 174.4 \\
\hline & $\mathrm{CH}_{2}-2$ & 2.28 & & 34.6 \\
\hline & $\mathrm{CH}_{2}-3$ & 1.58 & $\mathrm{~m}$ & 25.3 \\
\hline & $\mathrm{CH}_{2}$ & $1.28-1.22$ & $\mathrm{~m}$ & $29.6-32.0$ \\
\hline & $\mathrm{CH}_{2} \mathrm{n}-1$ & 1.26 & & 22.9 \\
\hline & $\mathrm{CH}_{3} \mathrm{n}$ & 0.84 & $\mathrm{t}$ & 14.4 \\
\hline \multirow[t]{3}{*}{ Diacylglycerol moiety } & $\mathrm{CH}-\mathrm{sn} 2$ & 5.06 & & 72.5 \\
\hline & $\mathrm{CH}-s n 1$ & $4.15,4.33$ & & 62.5 \\
\hline & CH-sn 3 & 3.65 & & 61.0 \\
\hline
\end{tabular}


Table 3. Cont.

\begin{tabular}{|c|c|c|c|c|}
\hline Compound & Assignment & ${ }^{1} \mathrm{H}(\mathrm{ppm})$ & $\begin{array}{l}\text { Multiplicity } \\
(\mathrm{J}(\mathrm{Hz}))\end{array}$ & ${ }^{13} \mathrm{C}(\mathrm{ppm})$ \\
\hline$\beta$-Sitosterol & $\mathrm{CH}_{3}-18$ & $0.66 *\left(\mathrm{I}_{\mathrm{STE}}\right)$ & $\mathrm{s}^{\mathrm{c}}$ & 12.2 \\
\hline \multirow[t]{5}{*}{ Squalene } & $\mathrm{CH}_{3}-\mathrm{a}$ & 1.56 & & 16.3 \\
\hline & $\mathrm{CH}_{3}-\mathrm{b}$ & 1.64 & & 25.8 \\
\hline & $\mathrm{CH}-\mathrm{c}$ & 5.07 & $\mathrm{~m}$ & 124.8 \\
\hline & $\mathrm{CH}_{2}-\mathrm{d}$ & 2.02 & & 27.4 \\
\hline & $\mathrm{CH}_{2}$-e & 1.96 & & 40.1 \\
\hline \multirow[t]{6}{*}{$\begin{array}{l}\text { 1,2-Diacyl-sn-glycero-3- } \\
\text { phosphatidylcholine }\end{array}$} & $\mathrm{N}\left(\mathrm{CH}_{3}\right)_{3}+$ & $3.22 *\left(\mathrm{I}_{\mathrm{PC}}\right)$ & $\mathrm{s}$ & 54.5 \\
\hline & $\mathrm{CH}_{2} \mathrm{~N}+$ & 3.75 & & 66.4 \\
\hline & $\mathrm{CH}_{2} \mathrm{OP}$ & 4.45 & & 60.6 \\
\hline & $\mathrm{CH}-\mathrm{sn} 2$ & 5.06 & & 72.5 \\
\hline & CH-sn 1 & $4.15,4.33$ & & 62.5 \\
\hline & $\mathrm{CH}-\mathrm{sn} 3$ & 3.65 & & 61.0 \\
\hline \multirow[t]{10}{*}{ Digalactosyldiacylglycerol } & $\mathrm{CH}^{\prime \prime}-1$ & $4.87 *\left(\mathrm{I}_{\mathrm{DGDG}}\right)$ & $\mathrm{d}^{\mathrm{d}}(3.8)$ & 99.8 \\
\hline & $\mathrm{CH}^{\prime \prime}-2$ & 3.77 & & 69.2 \\
\hline & $\mathrm{CH}^{\prime \prime}-3$ & 3.69 & & 70.6 \\
\hline & $\mathrm{CH}^{\prime \prime}-4$ & 3.91 & & 70.2 \\
\hline & $\mathrm{CH}^{\prime}-1$ & 4.19 & & 104.5 \\
\hline & $\mathrm{CH}^{\prime}-2,3$ & $3.51-3.53$ & & \\
\hline & $\mathrm{CH}^{\prime}-4$ & 3.90 & & \\
\hline & CH-sn 2 & 5.06 & & 72.5 \\
\hline & CH-sn 1 & $4.15,4.33$ & & 62.5 \\
\hline & $\mathrm{CH}-\mathrm{sn} 3$ & 3.65 & & 61.0 \\
\hline
\end{tabular}

${ }^{\mathrm{a}} \mathrm{m}=$ multiplet; ${ }^{\mathrm{b}} \mathrm{t}=$ triplet $^{\mathrm{c}} \mathrm{s}=$ singlet $^{\mathrm{d}} \mathrm{d}=$ doublet.

The amount of each quantified metabolite in organic extracts is reported in Table S2.

\subsection{Multivariate Statistical Analysis}

Principal component analysis (PCA) was carried out on 30 selected variables corresponding to 23 metabolites from the hydroalcoholic extract and 7 from the organic extract. In the case of fructose, glucose, and xylose, the sum of their alpha and beta anomeric forms instead of the individual isomer content was used in statistics. Before statistical analysis, the data were preprocessed using autoscaling: all of the variables were mean centered, and each variable was divided by its standard deviation. Principal component analysis was carried out using SIMCA software (version 12).

\section{Results}

\subsection{Assignments of Aqueous and Organic Extracts}

The ${ }^{1} \mathrm{H}$ and ${ }^{13} \mathrm{C}$ NMR spectra assignments apple aqueous (Figure 2) and organic (Figure 3) extracts of apple were carried out using 2D NMR experiments, standard compound addition, and literature data [24-26,37]. A more complete spectral assignment (Table 2) of the aqueous extracts with respect to literature data was obtained, identifying fructose tautomeric forms, galacturonic acid, GABA, and $p$-coumaroyl moiety. Fructose tautomeric forms, namely $\alpha$-D-fructofuranose and $\beta$-D-fructopyranose, were identified by means of their diagnostic ${ }^{1} \mathrm{H}$ NMR signals and $2 \mathrm{D}$ experiments. In particular, the presence of $\alpha$-D-fructofuranose was suggested by its characteristic signal at $4.13 \mathrm{ppm}$ due to the $\mathrm{CH}-3$ proton. ${ }^{1} \mathrm{H}_{-}{ }^{1} \mathrm{H}$ TOCSY experiment allowed us to identify the correlation with the $\mathrm{CH}-4$ proton (4.00 ppm), whereas the ${ }^{1} \mathrm{H}-{ }^{13} \mathrm{C}$ HMBC experiment showed the correlation of the $\mathrm{CH}-3$ proton with the $\mathrm{C}-2$ carbon at 105.5 ppm, typical of ketoses. Analogously, $\beta$-D-fructopyranose was recognized by the signal of the $\mathrm{CH}-5$ proton at $4.01 \mathrm{ppm}$, and the diagnostic spin system detected by the ${ }^{1} \mathrm{H}_{-}{ }^{1} \mathrm{H}$ TOCSY experiment allowed us to identify the other protons of this sugar, namely $\mathrm{CH}-3$ (3.80 ppm), $\mathrm{CH}-4$ (3.90 ppm), and $\mathrm{CH}_{2}-6,6^{\prime}$ $(3.72,4.03 \mathrm{ppm})$. C-2 carbon at $99.2 \mathrm{ppm}$ was also identified by means of the ${ }^{1} \mathrm{H}-{ }^{13} \mathrm{C}$ HMBC 
map. The presence of $\alpha$-galacturonic moiety was suggested by the diagnostic chemical shifts of the spin systems in the ${ }^{1} \mathrm{H}^{-1} \mathrm{H}$ TOCSY experiment, the coupling constants, and the correlations in the ${ }^{1} \mathrm{H}_{-}{ }^{13} \mathrm{C}$ HMBC experiment. In particular, the doublet at $4.41 \mathrm{ppm}$ with a J coupling constant of $1.2 \mathrm{~Hz}$ due to $\mathrm{CH}-5$ proton indicates single coupling between $\mathrm{H}-5$ and the equatorial $\mathrm{H}-4$. The ${ }^{1} \mathrm{H}-{ }^{1} \mathrm{H}$ TOCSY experiment allowed us to identify the other protons of this metabolite, namely CH-4 (4.29 ppm), CH-3 (3.90 ppm), CH-2 (3.80 ppm), and $\mathrm{CH}-1(5.31 \mathrm{ppm}$, doublet with $\mathrm{J}=3.80 \mathrm{~Hz})$. The correlation of the $\mathrm{CH}-5$ proton with carboxylic carbon at $177.1 \mathrm{ppm}$ was confirmed by the ${ }^{1} \mathrm{H}_{-}{ }^{13} \mathrm{C}$ HMBC map.
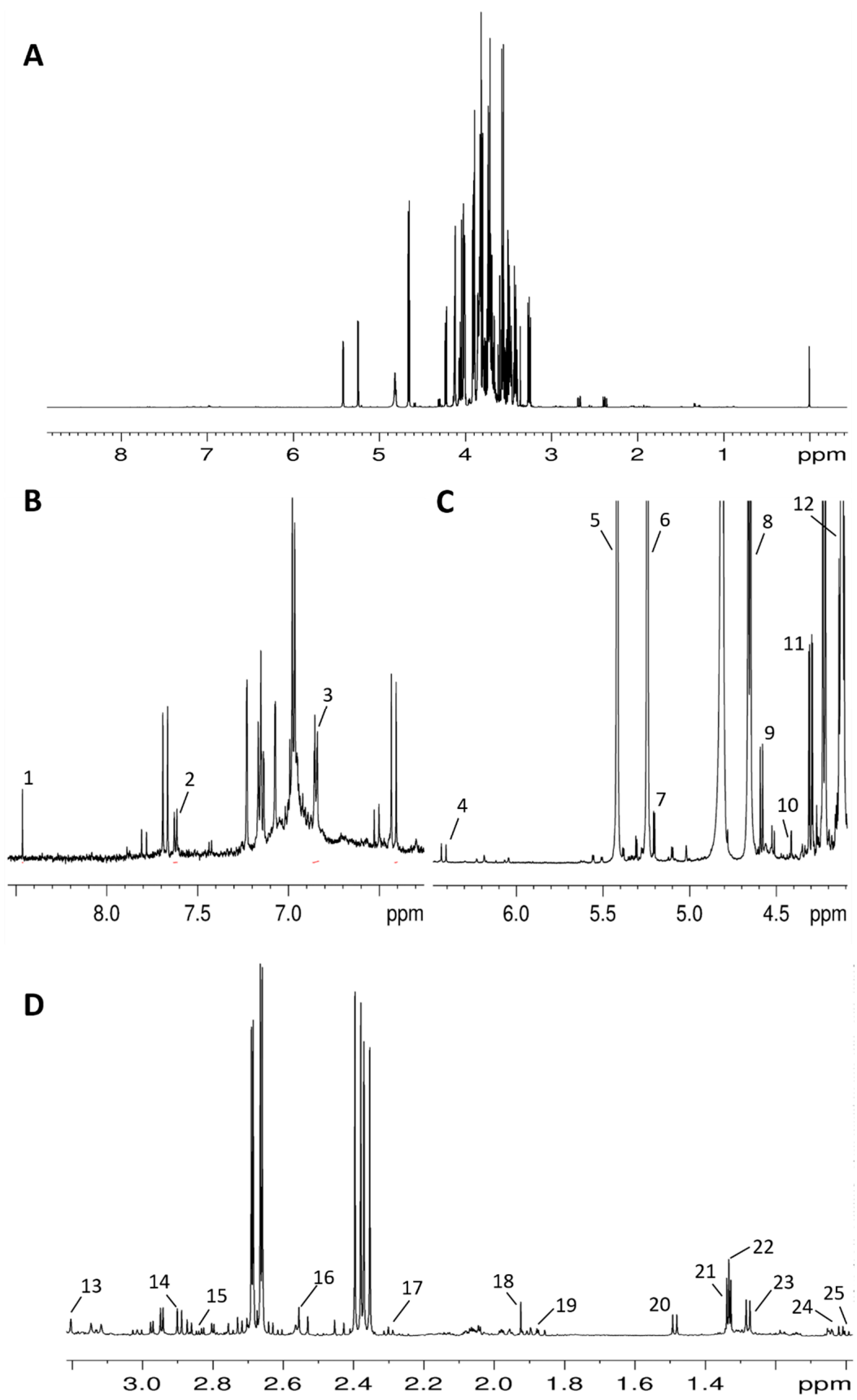

Figure 2. $600 \mathrm{MHz}{ }^{1} \mathrm{H}$ NMR spectrum of hydroalcoholic extract from apple fruit (var. Magnana) in a $400 \mathrm{mM}$ phosphate buffer (pH 7.4)/ $\mathrm{D}_{2} \mathrm{O}$ mixture with $2 \mathrm{mM}$ of 3-(trimethylsilyl)-propionic-2,2,3,3-d4 acid sodium salt (TSP). (A) Entire spectrum. (B) Low field region: 1, formic acid; 2, $p$-coumaric acid derivative; 3, phloretin/phloridzin. (C) Middle field region: 4 , chlorogenic acid; 5 , sucrose; 6 , $\alpha$-glucose; $7, \alpha$-xylose; $8, \beta$-glucose; $9, \beta$-xylose; $10, \alpha$-galacturonic acid; 11 , malic acid; 12 , D-fructofuranose, (D) High field region: 13, choline; 14, aspartate; 15, asparagine; 16, citric acid; 17, $\gamma$-aminobutyrate; 18, acetic acid; 19, quinic acid; 20, alanine; 21, lactic acid; 22, citramalic acid; 23, rhamnitol; 24, isoleucine; 25, valine. 

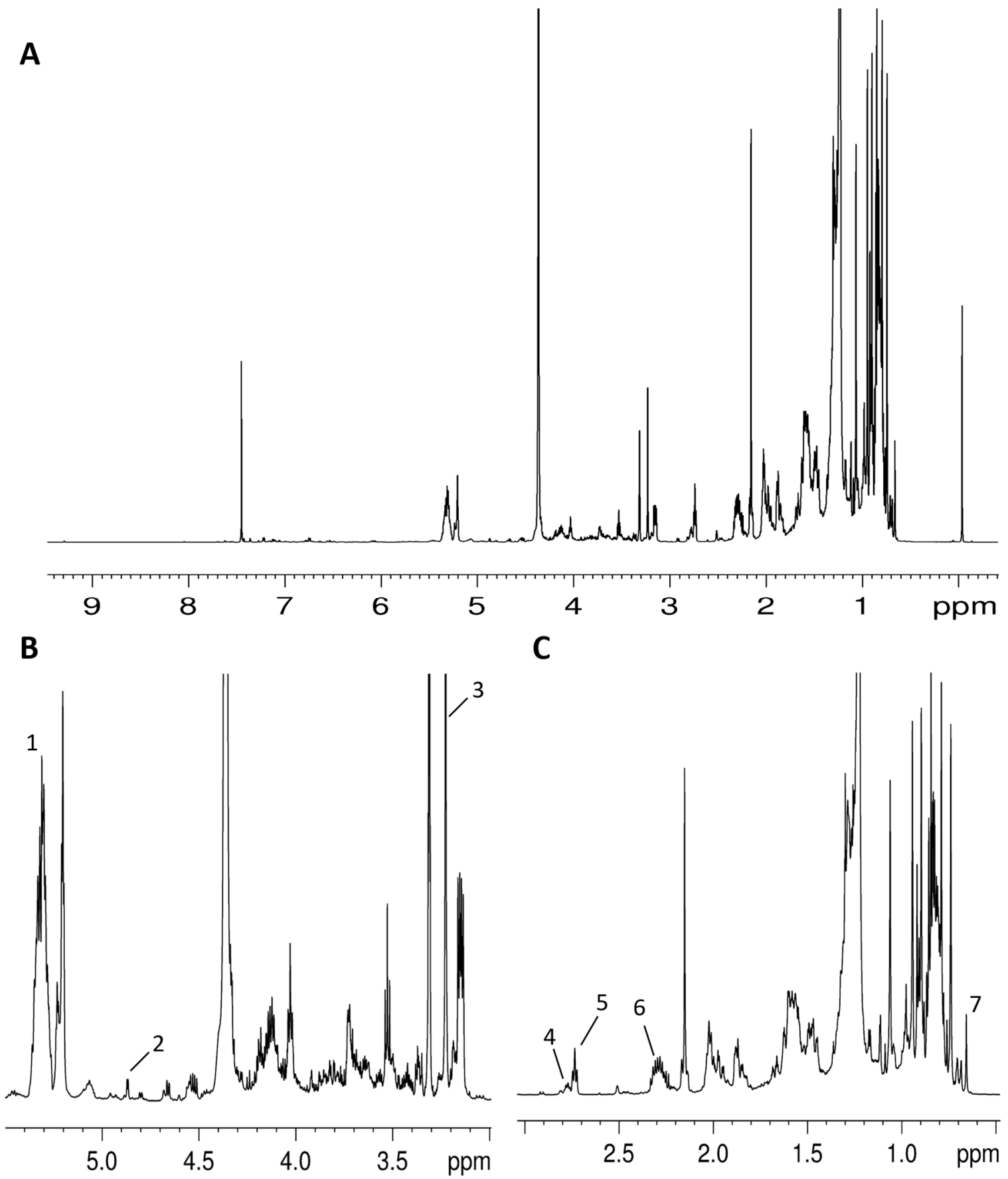

Figure 3. ${ }^{1} \mathrm{H}$ NMR spectrum at $600 \mathrm{MHz}$ of organic extract from apple fruit (var. Magnana) in the $\mathrm{CDCl}_{3} / \mathrm{CD}_{3} \mathrm{OH} 2: 1(v / v)$ mixture. (A) Entire spectrum. (B) Middle field region: 1, total unsaturated fatty acids; 2, digalactosyldiacylglycerol; 3 , 1,2-diacyl-sn-glycero-3-phosphatidylcholine. (C) High field region: 4, linolenic fatty chain; 5, linoleic fatty chain; 6, total fatty acids; $7, \beta$-sitosterol.

The presence of GABA was confirmed by its typical triplet at $2.30 \mathrm{ppm}(J=7.4 \mathrm{~Hz})$ assigned to $\alpha-\mathrm{CH}_{2}$ protons. The diagnostic spin systems identified in the ${ }^{1} \mathrm{H}-{ }^{1} \mathrm{H}$ TOCSY experiment allowed us to observe the correlations with $\beta-\mathrm{CH}_{2}$ and $\gamma-\mathrm{CH}_{2}$ protons at 1.90 and 3.01 ppm, respectively.

The diagnostic spin systems identified in the ${ }^{1} \mathrm{H}-{ }^{1} \mathrm{H}$ TOCSY experiment suggested the presence of $p$-coumaroyl moiety due to the correlation between $\mathrm{H}-2 / \mathrm{H}-6$ equivalent 
aromatic signals at $7.62 \mathrm{ppm}$ and $\mathrm{H}-3 / \mathrm{H}-5$ equivalent signals at $6.97 \mathrm{ppm}(\mathrm{J}=8.8 \mathrm{~Hz}$ corresponding to ortho position) and the correlation between the double bond protons at 7.79 and $6.51 \mathrm{ppm}$ with $J=16.1 \mathrm{~Hz}$ corresponding to trans configuration of the double bond. The low intensity of these signals-and, therefore, the low concentration of the corresponding compound-does not allow further correlations to be observed in the $2 \mathrm{D}$ experiments to complete the structural assignment. $p$-Coumaroyl quinic acid is the principal $p$-coumaroyl ester previously identified in apples using the targeted HPLC methodology $[13,38,39]$. Therefore, the identified $p$-coumaroyl moiety can be bound to quinic acid; however, the esterified quinic acid moiety was not detected due to the signal overlapping.

It was not possible to distinguish phloretin from its glycosides (such as phloridzin) using the available signals from aromatic protons region. Unfortunately, the signals expected for $\beta$-glucose moiety were not observed due to the strong overlapping with markedly more intense signals of other carbohydrates. The orto- and meta-protons of the para-substituted aromatic ring, a common moiety for all phloretin derivatives, gave rise to the signals at 7.16 and $6.85 \mathrm{ppm}$, respectively.

In Table 2, the assignment of four sugars (fructose, glucose, sucrose, and xylose), nine organic acids (acetic, citric, formic, citramalic, lactic, malic, quinic, and galacturonic acids), six amino acids (alanine, asparagine, aspartate, GABA, isoleucine, and valine), rhamnitol, p-coumaroyl derivative, phloretin/phloridzin, and choline is reported.

A more complete assignment of organic extracts of apples (Table 3) with respect to the literature data was obtained, reporting the assignment of glycerogalactolipid and glycerophospholipid polar heads. The presence of digalactosyldiacylglycerol (DGDG), the most abundant galactolipid in apples [40], was suggested by the characteristic doublet $(J=3.8 \mathrm{~Hz})$ at $4.87 \mathrm{ppm}$ due to the equatorial $\mathrm{CH}^{\prime \prime}-1$ proton of the external galactose ring [41]. The ${ }^{1} \mathrm{H}^{1}{ }^{1} \mathrm{H}$ TOCSY experiment allowed us to identify other protons of the ring, namely CH-2" (3.77 ppm), CH-3" (3.69 ppm), and CH-4" (3.91 ppm). Analogously, the $\mathrm{CH}-1^{\prime}$ proton of the internal DGDG galactose was also assigned at $4.19 \mathrm{ppm}$, together with the $\mathrm{CH}-2^{\prime} / \mathrm{CH}-3^{\prime}$ and $\mathrm{CH}-4^{\prime}$ protons (3.50-3.53 and $3.90 \mathrm{ppm}$, respectively).

Methyl groups of phosphatidylcholine $\mathrm{N}^{+}-\left(\mathrm{CH}_{3}\right)_{3}$ moiety were detected by the diagnostic ${ }^{1} \mathrm{H}$ and ${ }^{13} \mathrm{C}$ signals at $3.22 \mathrm{ppm}$ at $54.5 \mathrm{ppm}$, respectively. Moreover, the ${ }^{1} \mathrm{H}-{ }^{1} \mathrm{H}$ TOCSY experiment allowed us to identify proton correlation between the $\mathrm{CH}_{2} \mathrm{OP}$ group at $4.45 \mathrm{ppm}\left({ }^{13} \mathrm{C} 60.6 \mathrm{ppm}\right)$ and the $\mathrm{CH}_{2} \mathrm{~N}^{+}$protons at $3.75 \mathrm{ppm}\left({ }^{13} \mathrm{C} 66.4 \mathrm{ppm}\right)$.

In Table 3 , the assignment of $\beta$-Sitosterol, fatty acid chains, phosphatidylcholine, and digalactosyldiacylglycerol is also reported.

\subsection{Metabolite Profiles of Apple Cultivars}

In this section, each class of compounds is discussed separately, and a comparison of the ten cultivars is conducted.

\subsubsection{Sugars and Polyols}

The highest content of total sugars was measured in cultivars A9, A7, A3, and A2, whereas the lowest content was found in cultivars A1 and A6 (Figure 4). In particular, fructose, glucose and, sucrose were the most abundant sugars in all apple cultivars (Figure 5A). As expected [18], fructose turned out to be the main sugar in all samples, with the highest content detected in cultivars A5 and A7 and the lowest content in A1. The highest value of glucose content was measured in cultivar A2, whereas the lowest content ( more than three times less) was identified in sample A6. According to literature data [18,42,43], the fructose-to-glucose ratio was higher than 1.7 in all of the analyzed samples. Moreover, the fructose/glucose, fructose/sucrose, and sugar/acid weight ratios were calculated (Table 4). Cultivar A3 showed the highest sucrose content, whereas the lowest content was observed in A5 (more than four times less). Xylose was present in low concentrations in all of the cultivars, with cultivar A5 showing the highest content, whereas A1 showed the lowest content (more than six times). The highest content of rhamnitol, a polyol, was measured in cultivar A10, whereas the lowest content was found in A7. 
A

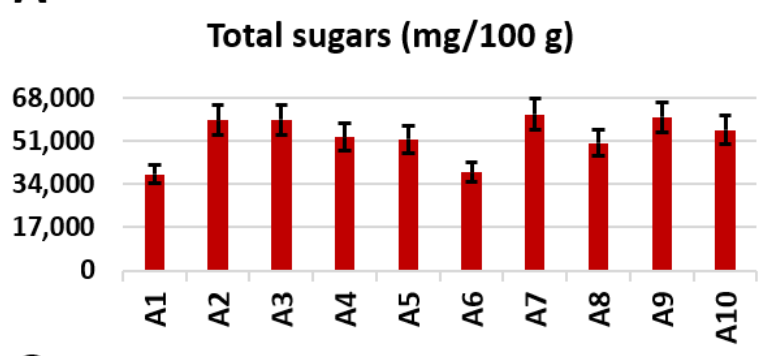

C

Total amino acids (mg/100 g)

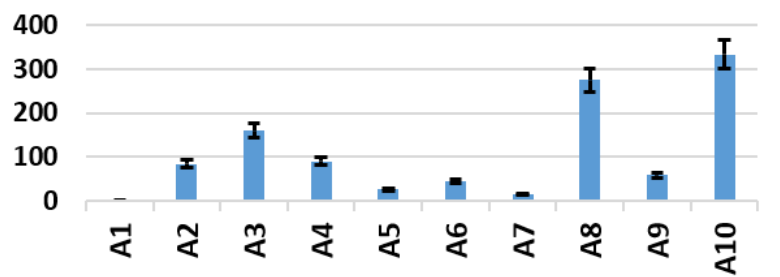

B

Total organic acids $(\mathrm{mg} / \mathbf{1 0 0} \mathrm{g})$

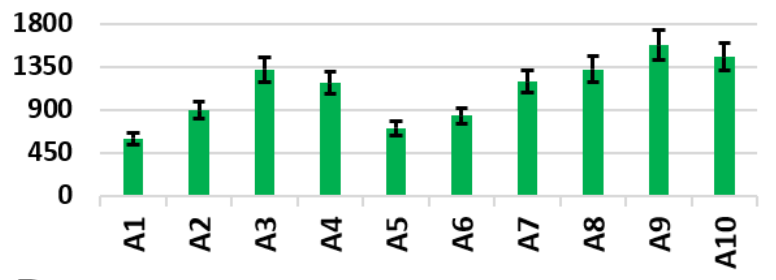

D

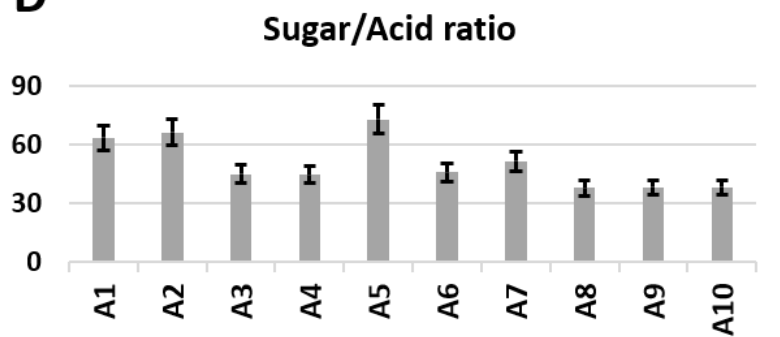

Figure 4. Bar charts of the total content of (A) sugars, (B) organic acids, (C) amino acids, (D) sugar/acid ratio, identified and quantified $(\mathrm{mg} / 100 \mathrm{~g}$ of $\mathrm{DW} \pm \mathrm{SD})$ in the ${ }^{1} \mathrm{H}$ NMR spectra of hydroalcoholic extracts of apples.

Table 4. Fructose/glucose, fructose/sucrose, and sugar/acid weight ratios in the ten studied Piedmont region apple cultivars and in three commercial cultivars (calculated using literature data).

\begin{tabular}{ccccc}
\hline Cultivar & Reference & Fru/Glc & Fru/Suc & Sugar/Acid \\
\hline Golden & {$[18]$} & 2.0 & 2.3 & 34 \\
Delicious & & & & \\
Golden & {$[44]$} & 2.9 & 1.8 & \\
Delicious & & & & 60 \\
Golden & {$[45]$} & 3.6 & 1.9 & 42 \\
Delicious & {$[18]$} & 1.7 & 2.8 & \\
Fuji & {$[44]$} & 15.0 & 2.0 & 30 \\
Fuji & {$[45]$} & 1.9 & 3.0 & 31 \\
Fuji & {$[18]$} & 7.7 & 2.6 & 127 \\
Jonagold & {$[44]$} & 3.2 & 1.1 & 57 \\
Jonagold & {$[45]$} & 2.3 & 1.8 & 61 \\
Jonagold & Present work & 1.8 & 2.4 & 44 \\
A1 & Present work & 3.6 & 3.2 & 43 \\
A2 & Present work & 3.5 & 1.3 & 69 \\
A3 & Present work & 2.9 & 6.1 & 42 \\
A4 & Present work & 4.2 & 2.0 & 49 \\
A5 & Present work & 3.6 & 2.1 & 37 \\
A6 & Present work & 3.8 & 2.3 & 37 \\
A7 & Present work & 4.3 & 1.5 & 38 \\
A8 & Present work & 3.2 & 1.3 & \\
A9 & Present work & & & \\
A10 & &
\end{tabular}



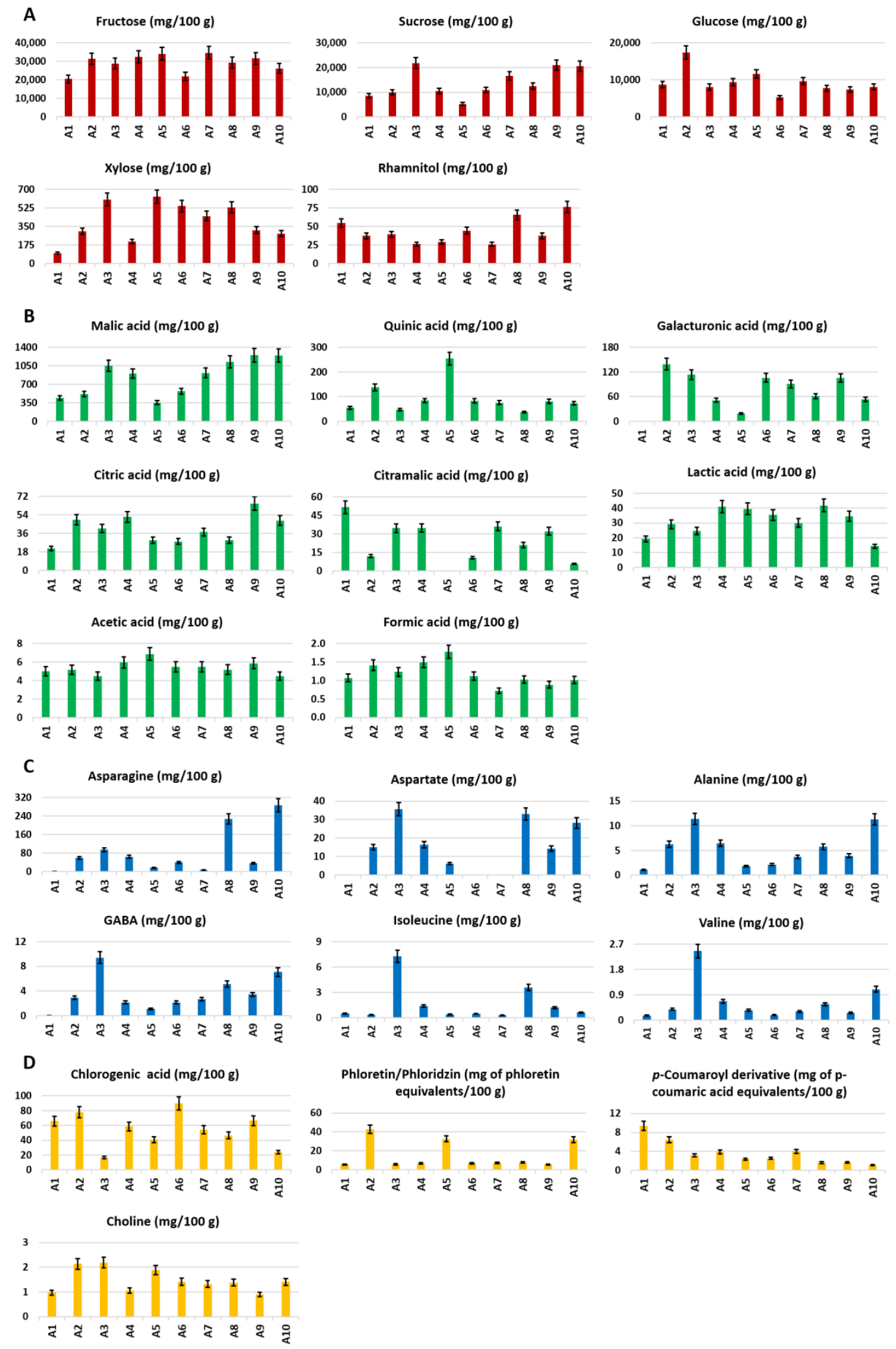

Figure 5. Bar charts of the metabolites identified and quantified (mg/100 $\mathrm{g}$ of $\mathrm{DW} \pm \mathrm{SD}$ ) in the ${ }^{1} \mathrm{H}$ NMR spectra of hydroalcoholic extracts of apples. (A) Sugars and polyols, (B) organic acids, (C) amino acids, (D) miscellaneous metabolites. Phloretin/phloridzin and $p$-coumaroyl derivative contents are expressed as phloretin equivalents and p-coumaric acid equivalents, respectively. 


\subsubsection{Organic Acids}

The highest total organic acids content (Figure 4) was found in cultivars A9 and A10, whereas the lowest amount was observed in cultivar A1 (more than two times less). In particular, malic acid was the most abundant acid in apples. The highest concentrations of malic acid were found in samples A9 and A10, whereas the lowest concentration was observed in A5 (Figure 5B). Cultivar A5 presented by far the highest content of quinic acid, whereas the lowest content (almost seven times less) was found in sample A8. The highest content of galacturonic acid was measured in cultivar A2, whereas it was not detected in sample A1. Cultivar A9 showed the highest amount of citric acid, whereas the lowest content was measured in A1 (more than three times less). Citramalic acid was not detected in sample A5; conversely, the highest amount was measured in sample A1. Cultivars A4 and A8 showed the highest content of lactic acid, whereas the lowest content was found in A10. Sample A10 was also characterized by the lowest amount of acetic acid, whereas cultivar A5 showed the highest amount. Finally, formic and acetic acids were found in very low concentrations, below $2 \mathrm{mg} / 100 \mathrm{~g}$.

\subsubsection{Amino Acids}

The content of free amino acids (total and those of individual components) was extremely variable among the different cultivars (Figure 5C). The highest total amino acid content was found in cultivars A8 and A10, whereas the lowest amount was observed in cultivars A1, A7, and A5 (Figure 4). In particular, cultivar A3 showed the highest amount of GABA, valine, and isoleucine, and, together with cultivar A10, alanine. Asparagine content varied from $280 \mathrm{mg} / 100 \mathrm{~g}$ in A10 to less than $1 \mathrm{mg} / 100 \mathrm{~g}$ (below the limit of detection) in A1. Aspartate was not found in cultivars A1, A6, or A7, and GABA was not detected in cultivar A1.

\subsubsection{Miscellaneous}

Cultivar A6 showed the highest amount of chlorogenic acid, whereas cultivar A3 the lowest amount (5 times less). A2, A5, and A10 showed by far the highest amounts of phloretin/phloridzin, whereas the lowest amount was observed in cultivar A1. The highest amount of $p$-coumaroyl derivatives was detected in cultivar A1, whereas the lowest amount was found in A10. The highest amount of choline was measured in cultivars A2 and A3, whereas the lowest amount was found in samples A1 and A9 (Figure 5D).

\subsection{5. $\beta$-Sitosterol}

This molecule was found in appreciable concentrations in all of the analyzed cultivars. In particular, the highest value was measured in cultivar A2, whereas the lowest amount was identified in A1 (Figure 6).

\subsubsection{Fatty Acids}

The content of unsaturated fatty acids (TOT UFA) was higher than the content of saturated fatty acids (TOT SFA) in all of the ten cultivars. Di-unsaturated fatty acids (DUFA) were the most abundant unsaturated fatty acids in all of the samples, the highest level being measured in cultivar A2 and the lowest in A1. Sample A1 was also characterized by the lowest value of mono-unsaturated fatty acids (MUFA) and the highest value of triunsaturated fatty acids (TUFA). Cultivar A4 showed an opposite trend, having the highest amount of MUFA and the lowest amount of TUFA. Finally, the highest concentration of TOT SFA was found in cultivar A1, whereas the lowest concentration was measured in sampleA8 (Figure 6). 


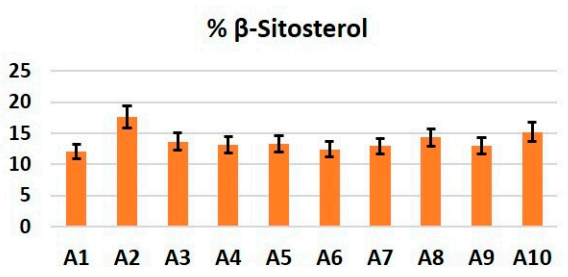

\% MUFA

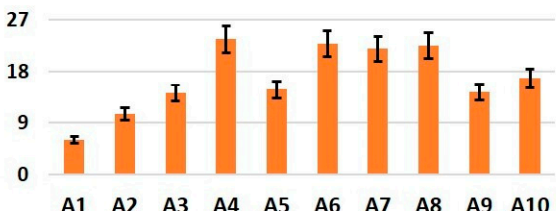

$\% \mathrm{PC}$

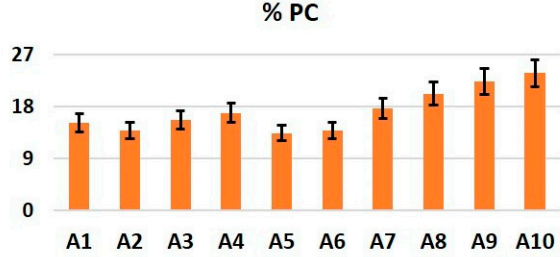

\% TOT SFA

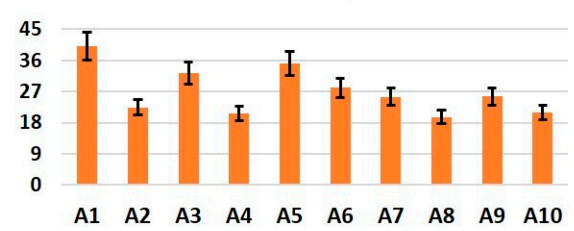

\% DUFA

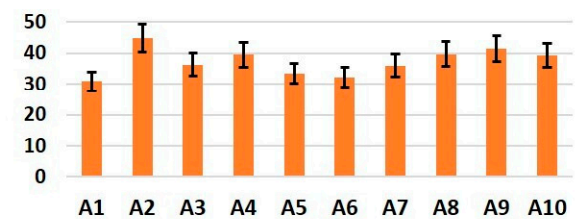

$\begin{array}{llllllllll}\text { A1 } & \text { A2 } & \text { A3 } & \text { A4 } & \text { A5 } & \text { A6 } & \text { A7 } & \text { A8 } & \text { A9 } & \text { A10 }\end{array}$

\% DGDG

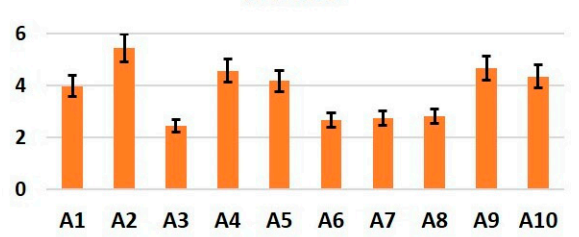

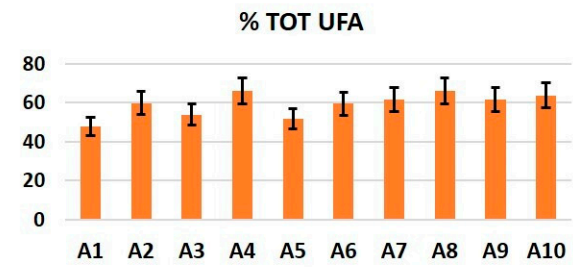

\% TUFA

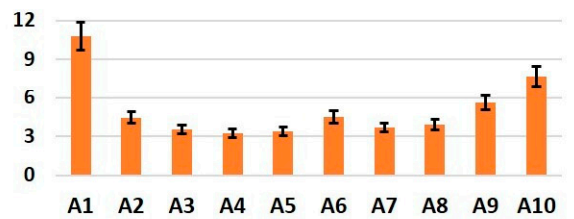

Figure 6. Bar charts of the metabolites (molar $\% \pm \mathrm{SD}$ ) identified and quantified in the ${ }^{1} \mathrm{H}$ NMR spectra of organic extracts of apples.

\subsubsection{Polar Lipids}

The highest content of phosphatidylcholine (PC) was observed in cultivar A10, whereas the lowest content was measured in A5. Cultivar A2 showed the highest concentration of digalactosyldiacylglycerol (DGDG), whereas the lowest content was observed in sample A3 (Figure 6).

\subsection{Multivariate Statistycal Analysis (PCA)}

The overall analysis of the histograms made it possible to find characteristic features relative to each cultivar. Moreover, PCA applied to all of the NMR variables allowed us to highlight possible cultivar similarities and differences (Figure 7).

Cultivar A1 (Canditina) was observed to be well separated from all of the others for the high content of citramalic acid, $p$-coumaroyl moiety, TUFA, and TOT SFA, and low amounts of xylose, fructose, sucrose, malic acid, citric acid, alanine, and MUFA. Notably, galacturonic acid, asparagine, aspartate, and GABA were not detected.

Carla, cultivar A5, was characterized by high contents of fructose, xylose, and organic acids (such as quinic acid, lactic acid, acetic acid, and formic acid) and low amounts of sucrose, chlorogenic acid, malic acid, amino acids (namely, asparagine aspartate, and alanine), and malic and galacturonic acids. Citramalic acid was not detected.

Gamba Fina, cultivar A6, was characterized by high content of MUFA and chlorogenic acid and low amounts of fructose, glucose, and citramalic acid. Aspartate was not detected.

Ross Giambon, cultivar A7, was characterized by high fructose and MUFA contents and low amounts of rhamnitol and asparagine. The level of aspartate was beyond the limit of detection. 
A

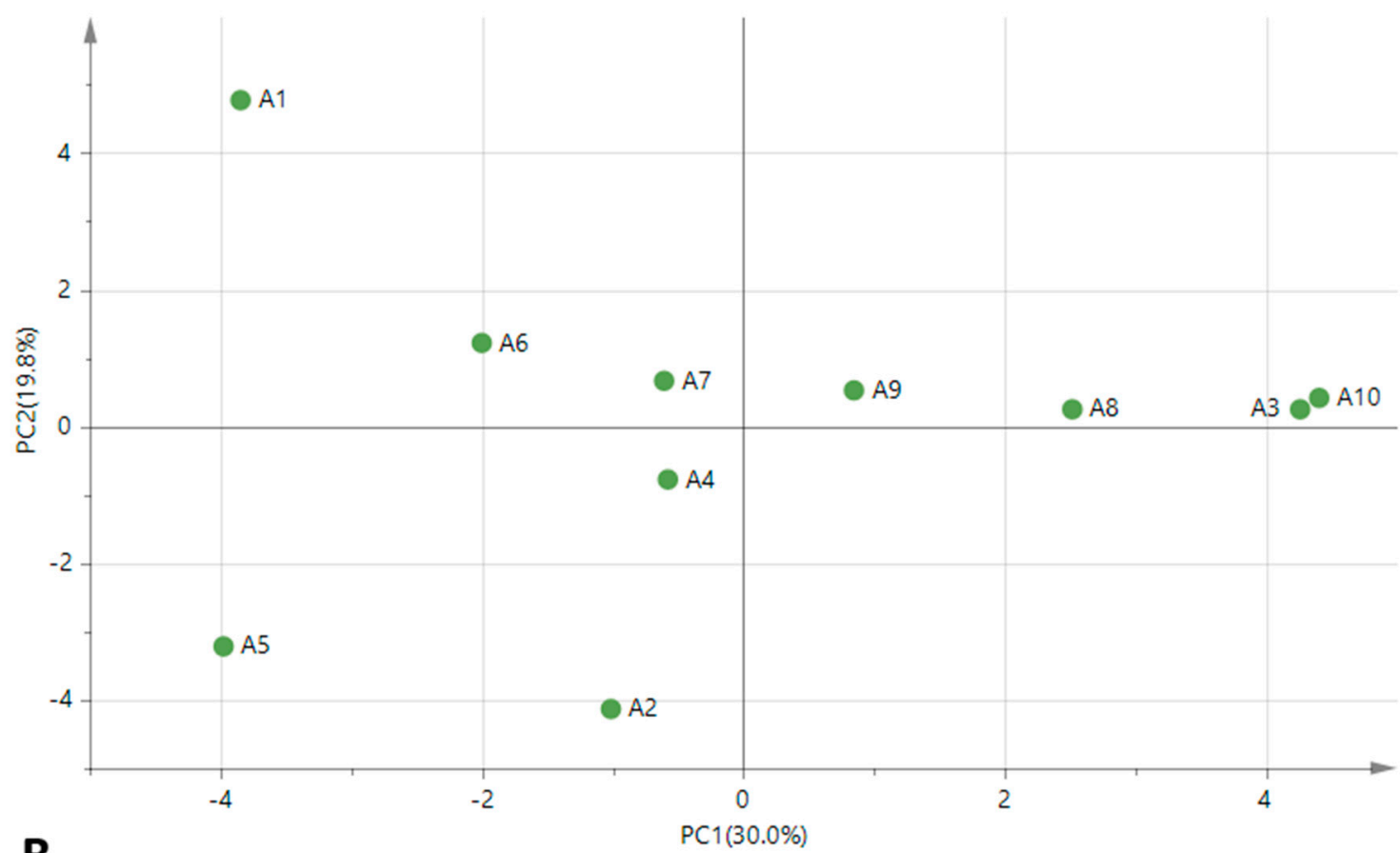

B

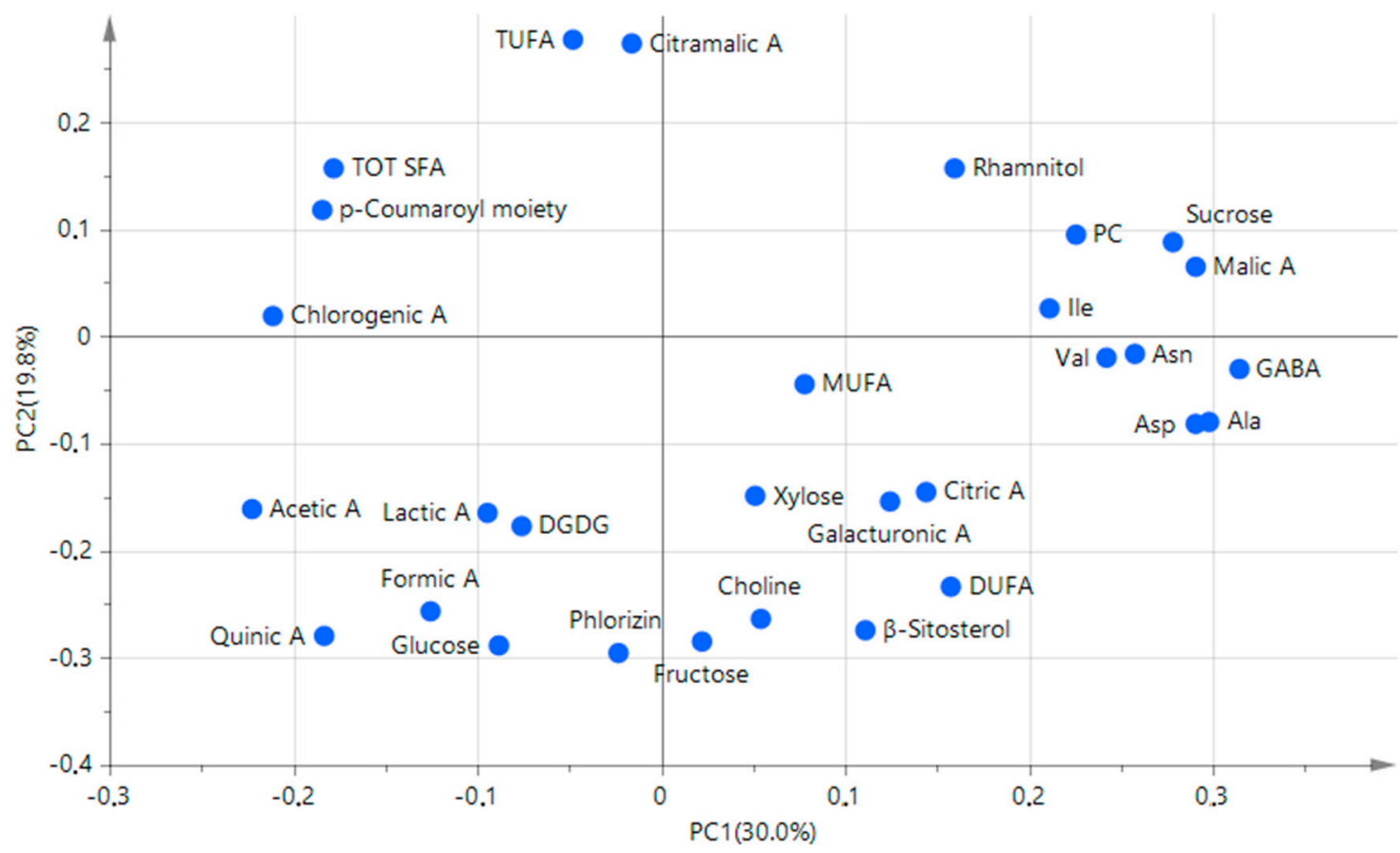

Figure 7. PCA applied to NMR data of the ten apple cultivars. (A) Sample scores and (B) loadings. PC1 and PC2 represent $30.0 \%$ and $19.8 \%$ of the total variance, respectively.

Runsé, cultivar A4, was characterized by high amounts of organic acids and MUFA.

Grigia di Torriana, cultivar A2, showed high amounts of glucose, galacturonic acid, choline, DUFA, and DGDG and a low level of citramalic acid.

Calvilla, cultivar A9, was characterized by high levels of sucrose, organic acids, DUFA and PC.

Dominici, cultivar A8, showed high contents of rhamnitol, lactic acid, asparagine, aspartate, isoleucine, and MUFA and a low concentration of quinic acid. 
The Magnana (A3) and Grenoble (A10) cultivars, situated nearby on the PCA score plot (PC1 > 4, Figure 3a), were characterized by high levels of sucrose, rhamnitol, malic acid, asparagine, alanine, and PC and low content of $p$-coumaroyl moiety.

\section{Discussion}

The present data on the chemical composition of traditional apple cultivars of the Piedmont region can be compared with those of some of the most widely cultivated apple cultivars in the world [46,47], such as Golden Delicious, Fuji, Granny Smith, and Jonagold $[18,44,45]$. Taking into account the fact that the data reported in the literature are related to apple juice composition (usually expressed as $\mathrm{g} / \mathrm{L}$ or $\mathrm{g} / \mathrm{kg} \mathrm{FW}$ ), while our data are related to dried tissue (expressed as $\mathrm{mg} / 100 \mathrm{~g}$ of $\mathrm{DW}$ ), it is clear that only the ratios between the components and not the absolute values can be directly compared.

The sugar content in apple fruit is very important, especially for diabetic patients who adapt their insulin intake in relation to the carbohydrate content of each food. Table 4 shows the ratios of fructose/glucose and fructose/sucrose calculated using the literature data $[18,44,45]$ for the most popular apple cultivars (Golden Delicious, Fuji, and Jonagold) in comparison with our data on Piedmont region cultivars. It is noteworthy that among three different studies of the same apple cultivars, there is a lack of agreement regarding sugar content. The studies only agree on the fact that fructose seemed to be the most abundant in all apple cultivars, whereas the relative content of glucose and sucrose was highly variable. The fructose/glucose ratio was always higher than 1.7 for all apple cultivars, whereas the upper limit can be as high as 15 (calculated for Fuji according to literature data [44]). In the case of Piedmont cultivars, the fructose/glucose ratio was in the range of 4.3 (A9)-1.8 (A2). The fructose/sucrose ratio was less variable, ranging from 1.1 (Jonagold, [44]) to 3.0 (Fuji, [45]). The corresponding values for Piedmont cultivars are inside this range, except for one (6.5), corresponding to cultivar A5 with the lowest sucrose content and a high fructose level.

The sugar-to-acid ratio is another important parameter indicating the taste and flavor of apples. In particular, a high level of the sugar/acid ratio is related to higher sweetness $[48,49]$. Again, the literature data on the sugar-to-acid ratio in Golden Delicious, Fuji, and Jonagold are not consistent-see Table 4. For example, for the Jonagold cultivar, values from 31 [18] to 127 [45] were reported. In our study, the cultivars with the highest sugar/acid ratio were Canditina (A1), Grigia di Torriana (A2), and Carla (A5) due to a low level of total organic acid, whereas a lower sugar/acid ratio was observed for the Dominici (A8), Calvilla (A9), and Grenoble (A10) cultivars.

The content of chlorogenic acid, one of the most abundant polyphenols in apple, has been reported to range from 34.9 (Golden Delicious) to $39.4 \mathrm{mg} / 100 \mathrm{~g}$ of DW (Jonagold) in the pulp of commercial cultivars [45]. Almost all Piedmont cultivars (except A3 and A10) were generally characterized by a higher content of chlorogenic acid with the maximum observed in Gamba Fina A6 (89 mg/100 g DW).

The fatty acid composition observed in the analyzed apple cultivars was similar to that of the literature data [18]. In particular, DUFA was by far the main class of fatty acids, followed by saturated fatty acids, MUFA, and TUFA. A high percentage of DUFA has been reported for Golden Delicious and Jonagold (more than $50 \mathrm{~mol} \%$ ) [11], whereas among the Piedmont cultivars, the highest DUFA content (45 mol\%) was observed in Canditina A1.

The identification of some characteristic metabolites of apples, such as phloridzin/ phloretin, rhamnitol, and citramalic acid, is particularly noteworthy. $[26,33,50,51]$ Their content is largely variable both in the analyzed cultivars and in others described in the literature $[18,25,26,52]$. These molecules are also widely studied to assign their role in the human body and in apple biosynthetic pathways.

Phloridzin as a polyphenol compound with antioxidant activity [53] shows some health benefits; in particular, it can be useful in the prevention of the type 2 diabetes mellitus [54,55] by reducing intestinal sugar uptake. Rhamnitol has been considered as a dietary biomarker in relation to the consumption of apples [56] and also as an important 
metabolite for the geographical discrimination of apple varieties with different geographical origins [26]. Finally, citramalic acid has been studied for its contribution to the development of anthocyanin in apple skin [57] and for its correlation with the storage of apples [58].

\section{Conclusions}

The presented results show that every local variety has its own chemical profile responsible for the sensorial, nutritional, and health-related properties, and they may be used as sources of specific substances that are utilized as ingredients of health products, such as food supplements, functional foods, and cosmetics. For instance, the Gamba Fina (A6) cultivar showed a significant content of chlorogenic acid with recognized properties against metabolic syndrome disorder [59,60], and the Grigia di Torriana (A2), Carla (A5), and Grenoble (A10) cultivars, with their high content of phloretin and phloridzin, could be considered very interesting for their possible properties against insulin resistance [54,55,61]. From a nutritional point of view, being rich in sugars, the Magnana cultivar (A3) can be used to prepare apple juice with no added sugars. Moreover, as the morphological characteristics (especially of the medium/small fruits) make these apple cultivars unattractive to the market for direct consumption, most of them could be successfully employed for the preparation of ingredients of nutraceutical products with high added value.

Although further studies should be performed to gain further understanding of the genetic and environmental basis that leads to these peculiar chemical compositions and the accumulation of polyphenols or nutrients, the reported data could be useful for national and international information systems to reinforce food and agriculture sectors, giving producers and industries accurate information regarding local food peculiarities [62].

Supplementary Materials: The following are available online at https:/ / www.mdpi.com/2304-8 158/10/2/289/s1, Table S1: Metabolite content in the hydroalcoholic extract of the analyzed apple cultivars from the Piedmont region $(\mathrm{mg} / 100 \mathrm{~g} \pm \mathrm{SD}$ ); Table S2: Metabolite content in the organic extract of the analyzed apple cultivars from the Piedmont region (molar \% $\pm \mathrm{SD}$ ).

Author Contributions: Conceptualization, L.M. and A.P.S.; methodology, M.S., G.D.M.; software, G.D.M.; validation, A.P.S., C.I. and L.M.; formal analysis, C.E., C.S.; investigation, M.S., G.D.M.; resources, C.I.; data curation, A.P.S.; writing—original draft preparation, M.S., G.D.M. and A.P.S.; writing-review and editing, M.D., A.B.; visualization, C.I.; supervision, A.P.S. and L.M. All authors have read and agreed to the published version of the manuscript.

Funding: This research was funded by Dipartimento di Chimica e Tecnologie del Farmaco and Italian Ministry of Education, Universities and Research-Dipartimenti di Eccellenza-L. 232/2016.

Data Availability Statement: The data presented in this study are available on request from the corresponding author.

Acknowledgments: The authors wish to thank "Azienda Agricola Melamangio", "Scuola Malva Arnaldi", and "Azienda Agricola Turaglio" for supplying apple samples, as well as Clara Bonifacio and Cinzia Pizzo for their contribution to the identification and collection of ancient cultivars.

Conflicts of Interest: The authors declare no conflict of interest. The funders had no role in the design of the study; in the collection, analyses, or interpretation of data; in the writing of the manuscript, or in the decision to publish the results.

\section{References}

1. FAO FAOSTAT Data. Available online: http:/ /www.fao.org/faostat/en/\#data/QC (accessed on 18 May 2020).

2. U.S. Department of Agriculture Food Data Central. Available online: https://fdc.nal.usda.gov/ (accessed on 18 May 2020).

3. Lee, K.W.; Kim, Y.J.; Kim, D.O.; Lee, H.J.; Lee, C.Y. Major Phenolics in Apple and Their Contribution to the Total Antioxidant Capacity. J. Agric. Food Chem. 2003, 51, 6516-6520. [CrossRef] [PubMed]

4. Francini, A.; Sebastiani, L. Phenolic compounds in apple (Malus x domestica borkh.): Compounds characterization and stability during postharvest and after processing. Antioxidants 2013, 2, 181-193. [CrossRef]

5. Wikiera, A.; Mika, M.; Grabacka, M. Multicatalytic enzyme preparations as effective alternative to acid in pectin extraction. Food Hydrocoll. 2015, 44, 156-161. [CrossRef] 
6. Güzel, M.; Akpınar, Ö. Valorisation of fruit by-products: Production characterization of pectins from fruit peels. Food Bioprod. Process. 2019, 115, 126-133. [CrossRef]

7. Caruso, T.; Ciarmiello, L.F.; Cutino, I.; Malvolti, M.E.; Murri, G.; Piccirillo, P. Atlante dei Fruttiferi Autoctoni Italiani; Crea-Centro di Frutticoltura: Roma, Italy, 2016; Volume III, pp. 1276-1285.

8. Arnaldi, S.M. Antiche Varietà Piemontesi, le Mele. Available online: https://www.antichevarietapiemontesi.it/mele/ (accessed on 6 August 2020).

9. Bounous, G. Antiche Cultivar di Melo in Piemonte. Piemonte, R., Ed.; 2006. Available online: http://hdl.handle.net/2318/15310 (accessed on 18 May 2020).

10. Bounous, G.; Melo, D.G.I. Collana Coltura \& Cultura; Script, A.R.T., Servizi, E.S.P.A., Eds.; Bayer Crop Science: Bologna, Italy, 2008; pp. 82-111.

11. Cerutti, A.K.; Bruun, S.; Donno, D.; Beccaro, G.L.; Bounous, G. Environmental sustainability of traditional foods: The case of ancient apple cultivars in Northern Italy assessed by multifunctional LCA. J. Clean. Prod. 2013, 52, 245-252. [CrossRef]

12. Donno, D.; Beccaro, G.L.; Mellano, M.G.; Torello Marinoni, D.; Cerutti, A.K.; Canterino, S.; Bounous, G. Application of sensory, nutraceutical and genetic techniques to create a quality profile of ancient apple cultivars. J. Food Qual. 2012, 35, 169-181. [CrossRef]

13. Ramirez-Ambrosi, M.; Abad-Garcia, B.; Viloria-Bernal, M.; Garmon-Lobato, S.; Berrueta, L.A.; Gallo, B. A new ultrahigh performance liquid chromatography with diode array detection coupled to electrospray ionization and quadrupole time-offlight mass spectrometry analytical strategy for fast analysis and improved characterization of phenolic compounds in ap. J. Chromatogr. A 2013, 1316, 78-91. [CrossRef]

14. Mari, A.; Tedesco, I.; Nappo, A.; Russo, G.L.; Malorni, A.; Carbone, V. Phenolic compound characterisation and antiproliferative activity of "Annurca" apple, a southern Italian cultivar. Food Chem. 2010, 123, 157-164. [CrossRef]

15. Guo, J.; Yue, T.; Yuan, Y.; Wang, Y. Chemometric Classification of Apple Juices According to Variety and Geographical Origin Based on Polyphenolic Profiles. J. Agric. Food Chem. 2013, 61, 6949-6963. [CrossRef]

16. Bai, L.; Guo, S.; Liu, Q.; Cui, X.; Zhang, X.; Zhang, L.; Yang, X.; Hou, M.; Ho, C.T.; Bai, N. Characterization of nine polyphenols in fruits of Malus pumila Mill by high-performance liquid chromatography. J. Food Drug Anal. 2016, 24, 293-298. [CrossRef]

17. Ferruzza, S.; Natella, F.; Ranaldi, G.; Murgia, C.; Rossi, C.; Trošt, K.; Mattivi, F.; Nardini, M.; Maldini, M.; Giusti, A.M.; et al. Nutraceutical improvement increases the protective activity of broccoli sprout juice in a human intestinal cell model of gut inflammation. Pharmaceuticals 2016, 9, 48. [CrossRef] [PubMed]

18. Wu, J.; Gao, H.; Zhao, L.; Liao, X.; Chen, F.; Wang, Z.; Hu, X. Chemical compositional characterization of some apple cultivars. Food Chem. 2007, 103, 88-93. [CrossRef]

19. Iaccarino, N.; Varming, C.; Petersen, M.A.; Viereck, N.; Schütz, B.; Toldam-Andersen, T.B.; Randazzo, A.; Engelsen, S.B. Ancient danish apple cultivars-A comprehensive metabolite and sensory profiling of apple juices. Metabolites 2019, 9, 139. [CrossRef] [PubMed]

20. Aprea, E.; Gika, H.; Carlin, S.; Theodoridis, G.; Vrhovsek, U.; Mattivi, F. Metabolite profiling on apple volatile content based on solid phase microextraction and gas-chromatography time of flight mass spectrometry. J. Chromatogr. A 2011, 1218, 4517-4524. [CrossRef] [PubMed]

21. Sobolev, A.P.; Thomas, F.; Donarski, J.; Ingallina, C.; Circi, S.; Cesare Marincola, F.; Capitani, D.; Mannina, L. Use of NMR applications to tackle future food fraud issues. Trends Food Sci. Technol. 2019, 91, 347-353. [CrossRef]

22. Belton, P.S.; Delgadillo, I.; Gil, A.M.; Roma, P.; Casuscelli, F.; Colquhoun, I.J.; Dennis, M.J.; Spraul, M. High-field proton NMR studies of apple juices. Magn. Reson. Chem. 1997, 35, 52-60. [CrossRef]

23. Berregi, I.; del Campo, G.; Caracena, R.; Miranda, J.I. Quantitative determination of formic acid in apple juices by $1 \mathrm{H}$ NMR spectrometry. Talanta 2007, 72, 1049-1053. [CrossRef] [PubMed]

24. Eisenmann, P.; Ehlers, M.; Weinert, C.H.; Tzvetkova, P.; Silber, M.; Rist, M.J.; Luy, B.; Muhle-Goll, C. Untargeted NMR spectroscopic analysis of the metabolic variety of new apple cultivars. Metabolites 2016, 6, 29. [CrossRef]

25. Sciubba, F.; Di Cocco, M.E.; Gianferri, R.; Capuani, G.; De Salvador, F.R.; Fontanari, M.; Gorietti, D.; Delfini, M. Nuclear Magnetic Resonance-Based Metabolic Comparative Analysis of Two Apple Varieties with Different Resistances to Apple Scab Attacks. J. Agric. Food Chem. 2015, 63, 8339-8347. [CrossRef]

26. Tomita, S.; Nemoto, T.; Matsuo, Y.; Shoji, T.; Tanaka, F.; Nakagawa, H.; Ono, H.; Kikuchi, J.; Ohnishi-Kameyama, M.; Sekiyama, Y. A NMR-based, non-targeted multistep metabolic profiling revealed l-rhamnitol as a metabolite that characterised apples from different geographic origins. Food Chem. 2015, 174, 163-172. [CrossRef]

27. Sobolev, A.P.; Mannina, L.; Capitani, D.; Sanzò, G.; Ingallina, C.; Botta, B.; Fornarini, S.; Crestoni, M.E.; Chiavarino, B.; Carradori, S.; et al. A multi-methodological approach in the study of Italian PDO “Cornetto di Pontecorvo" red sweet pepper. Food Chem. 2018, 255, 120-131. [CrossRef] [PubMed]

28. Ingallina, C.; Capitani, D.; Mannina, L.; Carradori, S.; Locatelli, M.; Di Sotto, A.; Di Giacomo, S.; Toniolo, C.; Pasqua, G.; Valletta, A.; et al. Phytochemical and biological characterization of Italian "sedano bianco di Sperlonga" Protected Geographical Indication celery ecotype: A multimethodological approach. Food Chem. 2020, 309, 125649. [CrossRef] [PubMed]

29. Ingallina, C.; Sobolev, A.P.; Circi, S.; Spano, M.; Giusti, A.M.; Mannina, L. New hybrid tomato cultivars: An NMR-based chemical characterization. Appl. Sci. 2020, 10, 1887. [CrossRef]

30. D'Imperio, M.; Dugo, G.; Alfa, M.; Mannina, L.; Segre, A.L. Statistical analysis on Sicilian olive oils. Food Chem. 2007, 102, 956-965. [CrossRef] 
31. Contessa, C.; Botta, R. Comparison of physicochemical traits of red-fleshed, commercial and ancient apple cultivars. Hortic. Sci. 2016, 43, 159-166. [CrossRef]

32. Cavanna, M.; Marinoni, D.T.; Bounous, G.; Botta, R. Genetic diversity in ancient apple germplasm from northwest Italy. J. Hortic. Sci. Biotechnol. 2008, 83, 549-554. [CrossRef]

33. Mannina, L.; Sobolev, A.P.; Viel, S. Liquid state 1H high field NMR in food analysis. Prog. Nucl. Magn. Reson. Spectrosc. 2012, 66, 1-39. [CrossRef]

34. Arnaldi, S.M. Piante di melo di Antiche Varietà piemontesi. Available online: http://www.scuolamalva.it/wp-content/uploads / 2016/06/Descrizione-piante-melo-TRIO.pdf (accessed on 6 August 2020).

35. Ingallina, C.; Sobolev, A.P.; Circi, S.; Spano, M.; Fraschetti, C.; Filippi, A.; Di Sotto, A.; Di Giacomo, S.; Mazzoccanti, G.; Gasparrini, F.; et al. Cannabis sativa L. Inflorescences from Monoecious Cultivars Grown in Central Italy: An Untargeted Chemical Characterization from Early Flowering to Ripening. Molecules 2020, 25, 1908. [CrossRef]

36. Zhu, D.; Shen, Y.; Wei, L.; Xu, L.; Cao, X.; Liu, H.; Li, J. Effect of particle size on the stability and flavor of cloudy apple juice. Food Chem. 2020, 328, 126967. [CrossRef]

37. Vermathen, M.; Marzorati, M.; Baumgartner, D.; Good, C.; Vermathen, P. Investigation of different apple cultivars by high resolution magic angle spinning NMR. A feasibility study. J. Agric. Food Chem. 2011, 59, 12784-12793. [CrossRef]

38. Vrhovsek, U.; Rigo, A.; Tonon, D.; Mattivi, F. Quantitation of polyphenols in different apple varieties. J. Agric. Food Chem. 2004, 52, 6532-6538. [CrossRef] [PubMed]

39. Panzella, L.; Petriccione, M.; Rega, P.; Scortichini, M.; Napolitano, A. A reappraisal of traditional apple cultivars from Southern Italy as a rich source of phenols with superior antioxidant activity. Food Chem. 2013, 140, 672-679. [CrossRef] [PubMed]

40. Sugawara, T.; Miyazawa, T. Separation and determination of glycolipids from edible plant sources by high-performance liquid chromatography and evaporative light-scattering detection. Lipids 1999, 34, 1231-1237. [CrossRef] [PubMed]

41. Ingallina, C.; Spano, M.; Sobolev, A.P.; Esposito, C.; Santarcangelo, C.; Baldi, A.; Daglia, M.; Mannina, L. Characterization of Local Products for Their Industrial Use : The Case of Italian Potato Cultivars Analyzed by Untargeted and Targeted Methodologies. Foods 2020, 9, 1216. [CrossRef] [PubMed]

42. Ticha, A.; Salejda, A.M.; Hyšpler, R.; Matejicek, A.; Paprstein, F.; Zadak, Z.; Cukrów, W.S. Jabłkach Różnych Odmian I Ich Wpływ Na Cechy Sensoryczne. Zywn. Nauk. Technol. Jakosc/Food. Sci. Technol. Qual. 2015, 22, 137-150. [CrossRef]

43. Hermann, K.; Bordewick-Dell, U. Fructose in different apple varieties. Implications for apple consumption in persons affected by fructose intolerance. Ernährungs Umschau 2018, 65, 48-52. [CrossRef]

44. Hecke, K.; Herbinger, K.; Veberič, R.; Trobec, M.; Toplak, H.; Štampar, F.; Keppel, H.; Grill, D. Sugar-, acid- and phenol contents in apple cultivars from organic and integrated fruit cultivation. Eur. J. Clin. Nutr. 2006, 60, 1136-1140. [CrossRef]

45. Kim, I.; Ku, K.H.; Jeong, M.C.; Kwon, S., II; Lee, J. Metabolite profiling and antioxidant activity of 10 new early- to mid-season apple cultivars and 14 traditional cultivars. Antioxidants 2020, 9, 443. [CrossRef]

46. Agricultural Marketing Resource Center Apples. Available online: https://www.agmrc.org/commodities-products/fruits/ apples (accessed on 6 August 2020).

47. Produce Report Global Trends in Apple Innovation. Available online: https://www.producereport.com/article/global-trendsapple-innovation (accessed on 6 August 2020).

48. Petkovsek, M.M.; Stampar, F.; Veberic, R. Parameters of inner quality of the apple scab resistant and susceptible apple cultivars (Malus domestica Borkh.). Sci. Hortic. 2007, 114, 37-44. [CrossRef]

49. Colaric, M.; Veberic, R.; Stampar, F.; Hudina, M. Evaluation of peach and nectarine fruit quality and correlations between sensory and chemical attributes. J. Sci. Food Agric. 2005, 85, 2611-2616. [CrossRef]

50. Hulme, A.C. The isolation of L-citramalic acid from the peel of the apple fruit. Biochim. Biophys. Acta 1954, 14, 36-43. [CrossRef]

51. Ehrenkranz, J.R.L.; Lewis, N.G.; Kahn, C.R.; Roth, J. Phlorizin: A review. Diabetes. Metab. Res. Rev. 2005, 21, 31-38. [CrossRef] [PubMed]

52. Escarpa, A.; González, M.C. High-performance liquid chromatography with diode-array detection for the determination of phenolic compounds in peel and pulp from different apple varieties. J. Chromatogr. A 1998, 823, 331-337. [CrossRef]

53. Boyer, J.; Liu, R.H. Apple phytochemicals and their health benefits. Nutr. J. 2004, 3, 1-15. [CrossRef]

54. Niederberger, K.E.; Tennant, D.R.; Bellion, P. Dietary intake of phloridzin from natural occurrence in foods. Br. J. Nutr. 2020, 123, 942-950. [CrossRef]

55. Kumar, S.; Sinha, K.; Sharma, R.; Purohit, R.; Padwad, Y. Phloretin and phloridzin improve insulin sensitivity and enhance glucose uptake by subverting PPAR $\gamma / \mathrm{Cdk} 5$ interaction in differentiated adipocytes. Exp. Cell Res. 2019, 383, 111480. [CrossRef]

56. Posma, J.M.; Garcia-Perez, I.; Heaton, J.C.; Burdisso, P.; Mathers, J.C.; Draper, J.; Lewis, M.; Lindon, J.C.; Frost, G.; Holmes, E.; et al. Integrated Analytical and Statistical Two-Dimensional Spectroscopy Strategy for Metabolite Identification: Application to Dietary Biomarkers. Anal. Chem. 2017, 89, 3300-3309. [CrossRef]

57. Noro, S.; Kudo, N.; Kitzuwa, T. Differences in Sugars and Organic Acids between Red and Yellow Apple Cultivars at Time of Coloring, and Effect of Citramalic Acid on Development of Anthocyanin. J. Jpn. Soc. Hortic. Sci. 1988, 57, 381-389. [CrossRef]

58. Rudell, D.R.; Mattheis, J.P.; Curry, E.A. Prestorage ultraviolet-white light irradiation alters apple peel metabolome. J. Agric. Food Chem. 2008, 56, 1138-1147. [CrossRef]

59. Santana-Gálvez, J.; Cisneros-Zevallos, L.; Jacobo-Velázquez, D.A. Chlorogenic Acid: Recent advances on its dual role as a food additive and a nutraceutical against metabolic syndrome. Molecules 2017, 22, 358. [CrossRef] 
60. Finamore, A.; Roselli, M.; Donini, L.M.; Brasili, D.E.; Rami, R.; Carnevali, P.; Mistura, L.; Pinto, A.; Giusti, A.M.; Mengheri, E. Supplementation with Bifidobacterium longum Bar33 and Lactobacillus helveticus Bar13 mixture improves immunity in elderly humans (over 75 years) and aged mice. Nutrition 2019, 63, 184-192. [CrossRef] [PubMed]

61. Boutaoui, N.; Zaiter, L.; Benayache, F.; Benayache, S.; Cacciagrano, F.; Cesa, S.; Secci, D.; Carradori, S.; Giusti, A.M.; Campestre, C.; et al. Atriplex mollis Desf. aerial parts: Extraction procedures, secondary metabolites and color analysis. Molecules 2018, 23, 1962. [CrossRef] [PubMed]

62. Toledo, Á.; Burlingame, B. Biodiversity and nutrition: A common path toward global food security and sustainable development. J. Food Compos. Anal. 2006, 19, 477-483. [CrossRef] 